FTUV/07-1103, IFIC/07-70, RM3-TH/07-16

\title{
Prospects for the Zee-Babu Model at the LHC and low energy experiments
}

\author{
Miguel Nebot \\ Centro de Física Teórica de Partículas (CFTP), \\ Instituto Superior Técnico, P-1049-001, Lisboa, Portugal and \\ Instituto Nazionale di Fisica Nucleare (INFN), Sezione di Roma Tre, \\ Dipartamento di Fisica "Edoardo Amaldi", \\ Università degli Studi Roma Tre, I-00146, Roma, Italy. \\ Josep F. Oliver, David Palao and Arcadi Santamaria \\ Departament de Física Teòrica, Universitat de València and \\ IFIC, Universitat de València-CSIC \\ Dr. Moliner 50, E-46100 Burjassot (València), Spain
}

\begin{abstract}
We analyze the viability of the Zee-Babu model as an explanation of observed neutrino masses and mixings and the possibility that the model is confirmed or discarded in experiments planned for the very close future. The allowed parameter space is studied analytically by using some approximations and partial data. Then, a complete scanning of all parameters and constraints is performed numerically by using Monte Carlo methods. The cleanest signal of the model will be the detection of the doubly charged scalar at the LHC and its correlation with measurements of the branching ratio of $\mu \rightarrow e \gamma$ at the MEG experiment. In addition, the model offers interesting predictions for $\tau^{-} \rightarrow \mu^{+} \mu^{-} \mu^{-}$experiments, lepton-hadron universality tests, the $\theta_{13}$ mixing in neutrino oscillations and the $\left\langle m_{\nu}\right\rangle_{e e}$ parameter of neutrinoless double beta decay.
\end{abstract}




\section{INTRODUCTION}

The hints for neutrino masses accumulated in the last 30 years [1, 2] have been converted into a strong evidence in the last 10 years [3, 4, 5, 6, 7, 8, 9, 10]: the only consistent explanation for solar neutrino data, atmospheric neutrino data, reactor and accelerator neutrino experiments is based on the hypothesis of massive neutrinos which mix and oscillate. Using this hypothesis all neutrino data can be fitted with just two squared mass differences and three mixing angles. At present, phases are not needed to explain the data although they can be included in the analyses and hopefully will be tested in the future.

This picture, although certainly a big step forward in our understanding of neutrino physics leaves more open questions than the standard model (SM) picture in which neutrinos are exactly massless because (1) the SM does not contain righthanded neutrinos, thus, Dirac neutrinos are not possible, and (2) the renormalizability of the model, with the minimal Higgs content, enforces the exact conservation of the lepton number ${ }^{1}$ which prevents Majorana mass terms. Therefore, to include neutrino masses, we should relax some or several of the above ingredients of the SM:

1. Add righthanded neutrinos.

2. Add new fields which could allow for lepton number violation while keeping the renormalizability of the model.

3. Drop the renormalizability of the model.

The last possibility is very general and allows for neutrino masses without touching the field content of the SM. However, its predictivity is very limited and it is only useful when seen as a low energy parametrization of a more complete theory containing heavy non-standard particles.

Allowing for righthanded neutrinos seems the simplest and most economical solution: if neutrinos are like all the other fermions, it is natural to consider righthanded neutrinos coupled to the lefthanded neutrinos and the Higgs scalar, obtaining in this way a Dirac mass

\footnotetext{
${ }^{1}$ Perturbatively both lepton $(L)$ and baryon $(B)$ numbers are conserved separately. If nonperturbative effects are taken into account only $B-L$ is exactly conserved. Still, $L$ and $B$ violations are tiny at zero temperature and density.
} 
term as all the other fermions have. One can then easily adjust these Yukawa couplings and fit all neutrino masses and mixings without problem.

However, neutrinos are not like all the other fermions: their masses are extremely small $m_{\nu}<1 \mathrm{eV}$. In addition, righthanded neutrinos are completely neutral with respect to the SM gauge group, therefore, nothing forbids a righthanded Majorana mass term in the SM. In fact, many will argue that it should be written unless a good reason is given to forbid it. If this mass is present and if it is very large, one can naturally explain the smallness of neutrino masses with the seesaw mechanism and perhaps provide a way to understand the amount of matter in the universe through the leptogenesis mechanism. Because of all these virtues, this model, SM plus heavy righthanded neutrinos, has almost universally become the standard mechanism for neutrino masses. However, the model is not completely satisfactory: the heavy Majorana neutrinos contribute, through loop effects, to the Higgs mass with terms which are proportional to the square of the righthanded Majorana mass. If this mass is huge, the contribution to the Higgs mass will be too large. This is a specific realization of the generic hierarchy problem of the SM. However, while within the strict SM one could argue that the new heavy particles contributing to the Higgs mass could have very small couplings to the Higgs boson, in the seesaw mechanism we have explicit particles with explicit masses and couplings giving contributions to the Higgs boson mass and the hierarchy problem cannot be swept under the rug anymore. Thus, supersymmetry or any other mechanism must be invoked to stabilize the Higgs boson mass. On the other hand, the fact that the righthanded neutrino mass is very large makes the effects of righthanded neutrinos negligible at low energies, except, of course, those related with neutrino oscillations. The model just provides neutrino masses and cannot be tested in present or planned experiments ${ }^{2}$.

Adding other fields to the SM gives many possibilities but obviously, if there are no righthanded neutrinos, all of them require the non-conservation of the lepton number and neutrinos acquire a Majorana mass term. Since supersymmetry provides the best solution to the hierarchy problem, one natural choice is to use the fields already present in the supersymmetric extensions of the SM to generate neutrino masses. Very interesting models of this type with spontaneous breakdown of R-parity (see for instance [11, 12]), which implies lepton number violation, or explicit R-parity violation (see [13, 14]) have been built. All

\footnotetext{
${ }^{2}$ Although one can check it indirectly through its effects in leptogenesis.
} 
these models basically use the sneutrinos to generate neutrino masses.

Alternatively one could add new ad hoc scalars to the SM in such a way that lepton number is not automatically conserved: triplets which develop a VEV [15, 16], charged singlets which could allow for explicit lepton number violation [17, 18, 19, 20] or a mixture of the two mechanisms (see for instance [21, 22]).

The seesaw mechanism is probably the most natural mechanism for neutrino masses. However, the LHC is going to provide results very soon and there are plans to increase the precision in $\mu \rightarrow e \gamma, \mu \rightarrow$ eee experiments in a couple of orders of magnitude (see for instance [23]). It may be time to explore alternatives for neutrino mass generation that could be confirmed or rejected in planned experiments; among them, the natural candidates are models in which neutrino masses are generated through radiative corrections: as the masses will be suppressed by loop effects the new particles responsible for them could be relatively light and be produced at the LHC, at the International Linear Collider (ILC) and may have sizable effects in $\mu \rightarrow e \gamma, \mu \rightarrow$ eee experiments. The simplest model of neutrino masses is the Zee-Babu ${ }^{3}$ (ZB) model [18, 19, 20] which just adds two complex singlet scalar fields to the SM (that is, just 4 new degrees of freedom) with neutrino masses generated at the two-loop level. Another very interesting model is the Zee model [17] which adds a new scalar doublet and a complex scalar singlet (6 new degrees of freedom), however, the simplest version of the model gives a too sharp bimaximal prediction for neutrino mixing and has already been excluded [24, 25, 26]. Therefore, we will only consider here the Zee-Babu model. In the Zee-Babu model neutrino masses are generated at two loops and are proportional to several Yukawa couplings of the new scalars and inversely proportional to the square of their masses, therefore the couplings cannot be too small and the scalar masses cannot be too large otherwise the generated neutrino masses would be too small. This is very interesting because the new scalars may be accessible at the LHC and could mediate the processes $\mu \rightarrow e \gamma$ and $\mu \rightarrow e e e$ with rates measurable in planned experiments. In this paper we will sharpen the predictions of the model by using both analytical and numerical methods, specially under the assumption that the new scalars are light enough to be produced at the LHC. The phenomenology of the Zee-Babu model was recently reviewed

\footnotetext{
3 The model was first proposed in [18, 19] and studied later in [20]. In the literature, it has been often referred to as the Babu model.
} 
in [27 $]^{4}$ where under certain assumptions analytic limits on several couplings and masses were set. The analysis of [27] is very clever and interesting, however, the calculation of several processes in that paper was taken from older papers with some wrong factors of 2 which, unfortunately, have also propagated to more recent papers. In addition, ref. [27] makes the simplifying assumption that certain couplings are negligible. Thus, we found interesting to review the phenomenology of the model by relaxing this assumption. This, unavoidably requires a numerical study which will be presented in this paper. We also take into account new stronger limits on flavour lepton number violating tau decays from BELLE [29, 30] and BABAR [31]. Thus, in section II] we sketch the model and review some of its features: the neutrino masses, contributions to low energy processes like $\mu \rightarrow e \gamma, \mu \rightarrow 3 e, \tau \rightarrow 3 \mu$ and favoured values for the parameters of the model. In section III we study the production and decays of the new scalars at the LHC. In section IV we analyze all the relevant constraints on the parameters of the model and obtain its predictions for lepton flavour violating processes and for neutrino mass parameters $\left(\theta_{13}\right.$ mixing, Majorana and Dirac phases, neutrinoless double beta decay parameter $\left.\left\langle m_{\nu}\right\rangle_{e e}, \ldots\right)$. Section $\mathrm{V}$ is devoted to a summary of the results. Finally in section $\mathrm{VI}$ we present our conclusions.

\section{THE ZEE-BABU MODEL}

The Zee-Babu model is the minimal extension of the SM providing neutrino masses and mixings compatible with experiment: in addition to the Standard Model field spectrum, it only contains one singly charged scalar and one doubly charged scalar.

In order to fix the notation we will briefly review the Zee-Babu model. We will denote the SM particle content as follows: $\ell$ will be the lefthanded lepton doublet, $\tilde{\ell} \equiv i \tau_{2} \ell^{c}=i \tau_{2} C \bar{\ell}^{T}$ is just the conjugate lepton doublet used to build Majorana type couplings, $e$ is the righthanded lepton and $H$ is the Higgs boson doublet. We also have, of course, the weak gauge bosons $\vec{W}_{\mu}$, $B_{\mu}$, and the quarks and gluons. As mentioned, the Zee-Babu model contains, in addition, two charged singlet scalar fields

$$
h^{ \pm}, \quad k^{ \pm \pm},
$$

with weak hypercharges \pm 1 and \pm 2 respectively. Here we will follow the convention $Q=$

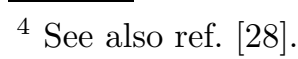


$T_{3}+Y$ and that $h$ and $k$ destroy negatively charged particles, thus $h=h^{-}$and $h^{\dagger}=h^{+}$, while $k=k^{--}$and $k^{\dagger}=k^{++}$. Then, the Lagrangian can be split into two parts:

$$
\mathcal{L}=\mathcal{L}_{\mathrm{SM}}+\mathcal{L}_{\mathrm{ZB}}
$$

The first part, $\mathcal{L}_{\mathrm{SM}}$, is the Standard Model Lagrangian:

$$
\mathcal{L}_{\mathrm{SM}}=i \bar{\ell} \not D \ell+i \bar{e} \not D e+(\bar{\ell} Y e H+\text { h.c. })+\cdots
$$

The dots represent SM gauge boson, Higgs boson and quark kinetic terms, quark Yukawa interactions and the SM Higgs potential. Generation and $S U(2)$ indices have been suppressed, and therefore, $Y$ is a completely general $3 \times 3$ matrix in generation space. The new terms in the Lagrangian are

$$
\mathcal{L}_{Z B}=D_{\mu} h^{\dagger} D^{\mu} h+D_{\mu} k^{\dagger} D^{\mu} k+\overline{\tilde{\ell}} f \ell h^{+}+\overline{e^{c}} g e k^{++}+\text {h.c. }-V_{Z B} .
$$

Since both $h$ and $k$ are $S U(2)$ singlets the covariant derivative only contains couplings to the $B$ gauge boson, which after diagonalization will generate photon and $Z$-boson couplings with the scalars, but no $W$ couplings. Due to Fermi statistics the Zee type Yukawa coupling, $f_{a b}$, is an antisymmetric matrix in flavour space while the Yukawa coupling, $g_{a b}$, of the doubly charged scalar $k$, is a symmetric matrix. The scalar potential $V_{Z B}$ contains all renormalizable interactions between the scalars $h, k$ and between them and the standard Higgs doublet:

$$
\begin{aligned}
V_{Z B} & =m_{h}^{\prime 2}|h|^{2}+m_{k}^{\prime 2}|k|^{2}+\lambda_{h}|h|^{4}+\lambda_{k}|k|^{4}+\lambda_{h k}|h|^{2}|k|^{2} \\
& +\lambda_{h H}|h|^{2} H^{\dagger} H+\lambda_{k H}|k|^{2} H^{\dagger} H+\left(\mu h^{2} k^{++}+\text {h.c. }\right) .
\end{aligned}
$$

Trilinear terms, like in the Zee model, with two scalar doublets and the $h$ vanish identically because the coupling is antisymmetric in $S U(2)$ indices and, therefore, requires two different doublets. The last term is particularly interesting because if $\mu \rightarrow 0$, the complete Lagrangian has an additional global $U(1)$ symmetry which can be identified with lepton number $L$ (or $B-L)$. In fact, if we assign lepton number 1 to both, the lepton doublet and the righthanded lepton singlet, we can also assign lepton number -2 to both, the scalars $h$ and $k$, in such a way that this quantum number is conserved in all the Lagrangian except in the trilinear coupling of the scalar potential. Thus, if $\mu \neq 0$, lepton number is explicitly broken by the $\mu$ coupling in the scalar potential. This is very important because this lepton number violation 
will be transmitted to the fermionic sector and will finally be responsible for the generation of neutrino masses. It is also important to remark that this mechanism for lepton number violation requires the simultaneous presence of the four couplings $Y, f, g$ and $\mu$, because if any of them vanishes one can always assign quantum numbers in such a way that there is a global $U(1)$ symmetry. This means that neutrino masses will require the simultaneous presence of the four couplings.

It is also important to note that the gauge-kinetic part of the Lagrangian is invariant under the following $U(N)$ transformations in generation space (for $N$ generations of leptons).

$$
\ell \rightarrow U_{\ell} \ell, \quad e \rightarrow U_{e} e
$$

Yukawa couplings, however, break this symmetry. This implies that sets of Yukawa couplings related by the following redefinitions are completely equivalent

$$
(Y, f, g) \rightarrow\left(U_{\ell}^{\dagger} Y U_{e}, U_{\ell}^{T} f U_{\ell}, U_{e}^{T} g U_{e}\right)
$$

which in turn means that physical observables should transform correctly under these redefinitions. This is important to check the behaviour under flavour transformations of physical amplitudes, moreover it can also be used to choose a convenient set of parameters in the Yukawa sector and count the number of physical parameters following the methods developed in [32]. Thus, using redefinitions of eq. (17) one can choose, without loss of generality, $Y$ diagonal with real and positive elements. One could also choose $f$ real and antisymmetric and leave $g$ as a completely general complex symmetric matrix. In addition, one can use redefinitions of $h^{+}$and $k^{++}$to set $\mu$ real and positive and to remove one of the phases in $g$. Thus, we finally have ${ }^{5} 12$ moduli ( 3 from $Y, 3$ from $f$ and 6 from $g$ ) and 5 phases (all from $g$ ) and the real and positive parameter $\mu$ (plus, of course, the rest of the parameters in the scalar potential). However, we will see later that this convention is not compatible with the standard parametrization of neutrino masses and mixings and it will be more convenient to use a slightly different convention for Yukawa coupling phases: we will also choose $Y$ diagonal with real and positive elements, then we will choose fermion field rephasings to remove 3 phases from the elements of $g_{a b}$, leaving the elements of $f_{a b}$ complex. Charged scalar rephasing can be further used to remove the phase of $\mu$ and one of the phases of $f_{a b}$,

\footnotetext{
${ }^{5}$ The counting can be generalized to $n$ generations of leptons. In that case we will have $n^{2}+n$ moduli and $n^{2}-n-1$ phases.
} 


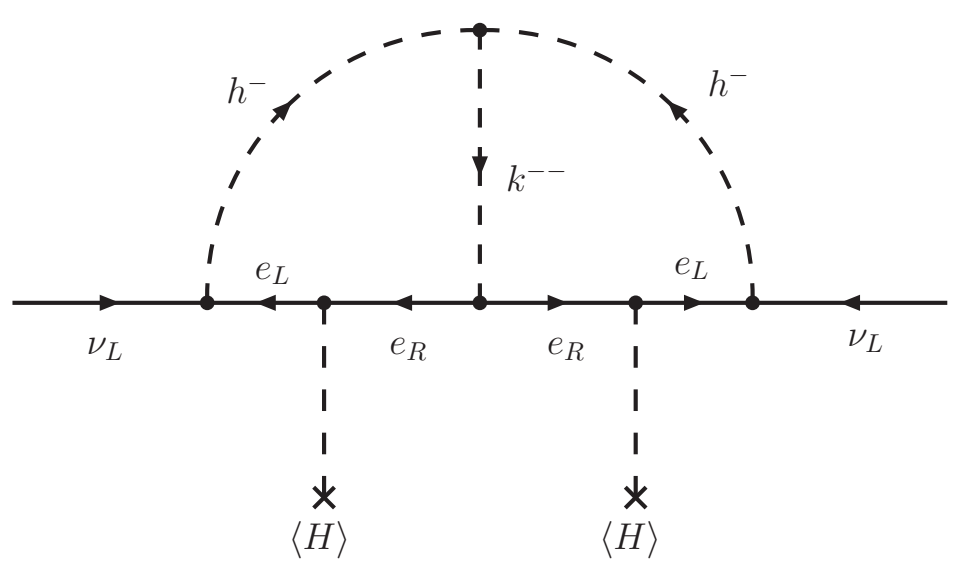

Figure 1: Diagram contributing to the neutrino Majorana mass at two loops.

for instance we can take $f_{\mu \tau}$ real and positive. Of course the counting of parameters is the same as before: we will have 12 moduli ( 3 from $Y, 3$ from $f$ and 6 from $g_{a b}$ ) and 5 phases (3 from $g_{a b}$ and 2 from $f_{a b}$ ) and the real and positive parameter $\mu$.

In any of the discussed conventions, $Y$ is directly related to the masses of charged leptons $m_{a}=Y_{a a} v$, with $v \equiv\left\langle H^{0}\right\rangle=174 \mathrm{GeV}$, the VEV of the standard Higgs doublet. Then the physical scalar masses are

$$
m_{h}^{2}=m_{h}^{\prime 2}+\lambda_{h H} v^{2}, \quad m_{k}^{2}=m_{k}^{\prime 2}+\lambda_{k H} v^{2}
$$

\section{A. The neutrino masses}

The first contribution to neutrino masses involving the four relevant couplings appears at two loops [19, 20] and its Feynman diagram is depicted in fig. 1.

The calculation of this diagram gives the following mass matrix for the neutrinos (defined as an effective term in the Lagrangian $\mathcal{L}_{\nu} \equiv-\frac{1}{2} \overline{\nu_{L}^{c}} \mathcal{M}_{\nu} \nu_{L}+$ h.c.)

$$
\left(\mathcal{M}_{\nu}\right)_{a b}=16 \mu f_{a c} m_{c} g_{c d}^{*} I_{c d} m_{d} f_{b d}
$$

with

$$
I_{c d}=\int \frac{\mathrm{d}^{4} k}{(2 \pi)^{4}} \int \frac{\mathrm{d}^{4} q}{(2 \pi)^{4}} \frac{1}{\left(k^{2}-m_{c}^{2}\right)} \frac{1}{\left(k^{2}-m_{h}^{2}\right)} \frac{1}{\left(q^{2}-m_{d}^{2}\right)} \frac{1}{\left(q^{2}-m_{h}^{2}\right)} \frac{1}{(k-q)^{2}-m_{k}^{2}} .
$$

$I_{c d}$ can be calculated analytically [33], however, since $m_{c}, m_{d}$ are the masses of the charged leptons, necessarily much lighter than the charged scalars, we can neglect them and obtain 
a much simpler form

$$
I_{c d} \simeq I=\frac{1}{\left(16 \pi^{2}\right)^{2}} \frac{1}{M^{2}} \frac{\pi^{2}}{3} \tilde{I}(r) \quad, \quad M \equiv \max \left(m_{h}, m_{k}\right),
$$

where $\tilde{I}(r)$ is a function of the ratio of the masses of the scalars $r \equiv m_{k}^{2} / m_{h}^{2}$,

$$
\tilde{I}(r)=\left\{\begin{array}{ll}
1+\frac{3}{\pi^{2}}\left(\log ^{2} r-1\right) & \text { for } r \gg 1 \\
1 & \text { for } r \rightarrow 0
\end{array},\right.
$$

which is close to one for a wide range of scalar masses. With this approximation the neutrino mass matrix can be directly written in terms of the Yukawa coupling matrices, $f, g$, and $Y$

$$
\mathcal{M}_{\nu}=\frac{v^{2} \mu}{48 \pi^{2} M^{2}} \tilde{I} f Y g^{\dagger} Y^{T} f^{T}
$$

A very important point is that since $f$ is a $3 \times 3$ antisymmetric matrix, $\operatorname{det} f=0$, and therefore $\operatorname{det} \mathcal{M}_{\nu}=0$. Thus, at least one of the neutrinos is exactly massless at this order ${ }^{6}$. This is a very important result since it excludes the possibility of degenerate neutrino masses.

To estimate the value of the largest possible neutrino mass we can take $\mu \approx m_{k} \approx m_{h} \equiv$ $M$, then the largest $\nu$ mass will be

$$
m_{\nu} \approx 6.6 \times 10^{-3} f^{2} g \frac{m_{\tau}^{2}}{M}
$$

which is the typical seesaw formula, suppressed by some additional couplings and loop factors. Because in this model one of the neutrinos is massless, the heaviest neutrino mass is fixed by the atmospheric mass difference, thus $m_{\nu} \approx 0.05 \mathrm{eV}$ and

$$
f^{2} g \approx 150 \frac{M m_{\nu}}{m_{\tau}^{2}}>2 \times 10^{-7}
$$

since LEP bounds on charged scalar masses are typically $M>100 \mathrm{GeV}$. This means that $f$ 's and $g$ 's cannot be made arbitrarily small and natural values for them can be $g, f \gtrsim 0.01$. Then, for these relatively large couplings and scalar masses in a range $M \sim 100 \mathrm{GeV}-10 \mathrm{TeV}$ the model will give sizable contributions to low energy processes like $\mu \rightarrow e e e, \mu \rightarrow e \gamma, \ldots$, and scalars that could be produced and detected at the LHC.

\footnotetext{
${ }^{6}$ This result does not change if higher orders in charged leptons masses are taken into account in the loop integral $I_{a b}$. However, one expects it will change if higher loops are considered.
} 
Alternatively, if we assume that the Yukawa couplings are smaller than one, $f, g<1$ we could write

$$
M \approx 6.6 \times 10^{-3} f^{2} g \frac{m_{\tau}^{2}}{m_{\nu}}<4 \times 10^{5} \mathrm{TeV},
$$

which is out of reach of planned experiments. However, constraints on low energy processes might require smaller couplings which would lead to much smaller scalar masses. In addition, these estimates are very rough; for example the relevant couplings may be related to muon physics, and not to tau physics: our estimate on neutrino masses should then be reduced by a factor $\left(m_{\mu} / m_{\tau}\right)^{2}$, which of course requires much lighter scalars to match the atmospheric neutrino scale. It is therefore very important to carefully establish the parameters and validity of the model.

\section{B. Low energy constraints}

In order to provide neutrino masses compatible with experiment, the Yukawa couplings of the charged scalars cannot be too small and their masses cannot be too large. This immediately gives rise to a series of flavour lepton number violating processes, as for instance $\mu^{-} \rightarrow e^{-} \gamma$ or $\mu^{-} \rightarrow e^{+} e^{-} e^{-}$, with rates which can be, in some cases, at the verge of the present experimental limits. This means that we can use these processes to obtain information on the parameters of the model and perhaps to confirm or to exclude the model in a close future. In this section we will discuss briefly the relevant processes and collect the formulas for our conventions of Yukawa couplings:

- $\ell_{a}^{-} \rightarrow \ell_{b}^{+} \ell_{c}^{-} \ell_{d}^{-}:$The interesting observable for these processes is the decay width. We have (see for instance [34])

$$
\mathrm{R}\left(\ell_{a}^{-} \rightarrow \ell_{b}^{+} \ell_{c}^{-} \ell_{d}^{-}\right) \equiv \frac{\Gamma\left(\ell_{a}^{-} \rightarrow \ell_{b}^{+} \ell_{c}^{-} \ell_{d}^{-}\right)}{\Gamma\left(\ell_{a}^{-} \rightarrow \ell_{b}^{-} \nu \bar{\nu}\right)}=\frac{1}{2\left(1+\delta_{c d}\right)}\left|\frac{g_{a b} g_{c d}^{*}}{G_{F} m_{k}^{2}}\right|^{2} .
$$

In this expression, the term $\delta_{c d}$ takes into account the fact that we may have two identical particles in the final state. In the case of $\tau$ decays we have to remember that leptonic channels are a small fraction of the decays $\operatorname{BR}\left(\ell_{a}^{-} \rightarrow \ell_{b}^{+} \ell_{c}^{-} \ell_{d}^{-}\right)=$ $\mathrm{R}\left(\ell_{a}^{-} \rightarrow \ell_{b}^{+} \ell_{c}^{-} \ell_{d}^{-}\right) \operatorname{BR}\left(\ell_{a}^{-} \rightarrow \ell_{b}^{-} \nu \bar{\nu}\right)\left(\right.$ with $\operatorname{BR}\left(\mu^{-} \rightarrow e^{-} \nu \bar{\nu}\right) \approx 100 \%, \operatorname{BR}\left(\tau^{-} \rightarrow e^{-} \nu \bar{\nu}\right) \approx$ $17.84 \%$ and $\left.\operatorname{BR}\left(\tau^{-} \rightarrow \mu^{-} \nu \bar{\nu}\right) \approx 17.36 \%\right)$. 


\begin{tabular}{ccl}
\hline \multicolumn{1}{c}{ Process } & Experiment $(90 \% \mathrm{CL})$ & Bound $(90 \% \mathrm{CL})$ \\
\hline$\mu^{-} \rightarrow e^{+} e^{-} e^{-}$ & $\mathrm{BR}<1.0 \times 10^{-12}$ & $\left|g_{e \mu} g_{e e}^{*}\right|<2.3 \times 10^{-5}\left(m_{k} / \mathrm{TeV}\right)^{2}$ \\
$\tau^{-} \rightarrow e^{+} e^{-} e^{-}$ & $\mathrm{BR}<3.6 \times 10^{-8}$ & $\left|g_{e \tau} g_{e e}^{*}\right|<0.010\left(m_{k} / \mathrm{TeV}\right)^{2}$ \\
$\tau^{-} \rightarrow e^{+} e^{-} \mu^{-}$ & $\mathrm{BR}<2.7 \times 10^{-8}$ & $\left|g_{e \tau} g_{e \mu}^{*}\right|<0.006\left(m_{k} / \mathrm{TeV}\right)^{2}$ \\
$\tau^{-} \rightarrow e^{+} \mu^{-} \mu^{-}$ & $\mathrm{BR}<2.3 \times 10^{-8}$ & $\left|g_{e \tau} g_{\mu \mu}^{*}\right|<0.008\left(m_{k} / \mathrm{TeV}\right)^{2}$ \\
$\tau^{-} \rightarrow \mu^{+} e^{-} e^{-}$ & $\mathrm{BR}<2.0 \times 10^{-8}$ & $\left|g_{\mu \tau} g_{e e}^{*}\right|<0.008\left(m_{k} / \mathrm{TeV}\right)^{2}$ \\
$\tau^{-} \rightarrow \mu^{+} e^{-} \mu^{-}$ & $\mathrm{BR}<3.7 \times 10^{-8}$ & $\left|g_{\mu \tau} g_{e \mu}^{*}\right|<0.008\left(m_{k} / \mathrm{TeV}\right)^{2}$ \\
$\tau^{-} \rightarrow \mu^{+} \mu^{-} \mu^{-}$ & $\mathrm{BR}<3.2 \times 10^{-8}$ & $\left|g_{\mu \tau} g_{\mu \mu}^{*}\right|<0.010\left(m_{k} / \mathrm{TeV}\right)^{2}$ \\
$\mu^{+} e^{-} \rightarrow \mu^{-} e^{+}$ & $G_{M \bar{M}}<0.003 G_{F}$ & $\left|g_{e e} g_{\mu \mu}^{*}\right|<0.2\left(m_{k} / \mathrm{TeV}\right)^{2}$ \\
\hline
\end{tabular}

Table I: Constraints from tree-level lepton flavour violating decays.

- $\mu^{+} e^{-} \longleftrightarrow \mu^{-} e^{+}$: The $k^{++}$scalar exchange gives also rise to transitions of the type $\mu^{+} e^{-} \rightarrow \mu^{-} e^{+}$which are well bounded experimentally. The relevant four-fermion effective coupling generated by exchange of the scalar $k^{++}$is (here we use the conventions for the effective Hamiltonian and the limits of [35, 36] )

$$
G_{M \bar{M}}=-\frac{\sqrt{2}}{8} \frac{g_{e e} g_{\mu \mu}^{*}}{m_{k}^{2}} .
$$

We collect the relevant constraints of this type in table $\llbracket$.

- $\ell_{a} \rightarrow \ell_{b} \nu \bar{\nu}:$ These processes receive additional contributions from the exchange of the singly charged scalar $h^{+}$which affect the Fermi muon decay constant but do not modify the spectrum [21]

$$
\left(\frac{G_{\mu}}{G_{\mu S M}}\right)^{2} \approx 1+\frac{\sqrt{2}}{G_{F} m_{h}^{2}}\left|f_{e \mu}\right|^{2}+\frac{1}{2 G_{F}^{2} m_{h}^{4}}\left(\left|f_{e \mu}\right|^{2}+\left|f_{e \tau}\right|^{2}\right)\left(\left|f_{e \mu}\right|^{2}+\left|f_{\mu \tau}\right|^{2}\right)
$$

where a sum over undetected neutrinos has been performed. The second term is clearly subdominant if $m_{h} \gg 200 \mathrm{GeV}$, however we have included it in the numerical analysis and have checked that we can neglect it in analytical estimates. In this model the charged scalar only contributes to lepton decays but does not contribute to hadronic decays, therefore the effective $G_{\beta}$ extracted from hadronic decays and $G_{\mu}$ are different. However, in the framework of the SM, the equality of $G_{\beta}$ and $G_{\mu}$ has been tested with very good accuracy once all radiative corrections have been correctly included. In the SM, both $\left|V_{u d}\right|^{2}+\left|V_{u s}\right|^{2}+\left|V_{u b}\right|^{2}=1$ and $G_{\beta S M}=G_{\mu S M}$ are satisfied. Thus, assuming 
$G_{\beta S M}=G_{\mu S M}$ one can test the unitarity of the CKM matrix, or, conversely, assuming the unitarity of the CKM matrix one can test the universality of couplings in hadronic and leptonic decays. In the model we are considering this is not true anymore; the CKM matrix is still unitary but, as explained, $G_{\beta}=G_{\beta S M}=G_{\mu S M} \neq G_{\mu}$. Since the extraction of the experimental values of $\left|V_{i j}^{e x p}\right|^{2}$ assumes the SM, we will have

$$
V_{i j}^{e x p}=\frac{G_{\beta}}{G_{\mu}} V_{i j},
$$

where $V_{i j}$ are the truly unitary CKM matrix elements in the model. Therefore

$$
\left|V_{u d}^{e x p}\right|^{2}+\left|V_{u s}^{e x p}\right|^{2}+\left|V_{u b}^{e x p}\right|^{2}=\frac{G_{\beta}^{2}}{G_{\mu}^{2}}=\frac{G_{\mu S M}^{2}}{G_{\mu}^{2}} \approx 1-\frac{\sqrt{2}}{G_{F} m_{h}^{2}}\left|f_{e \mu}\right|^{2},
$$

and, since $\left|V_{u d}^{e x p}\right|^{2}+\left|V_{u s}^{e x p}\right|^{2}+\left|V_{u b}^{e x p}\right|^{2}=0.9992 \pm 0.0011$ [37] is very close to 1 , we will obtain a strong limit on $\left|f_{e \mu}\right|^{2} / m_{h}^{2}$.

On the other hand, the charged scalar contribution will also modify the Fermi coupling extracted from $\tau$ decays in the different leptonic channels. After subtracting the different factors from phase space and radiative corrections this is usually expressed in terms of ratios of effective "gauge couplings" $g_{a}^{e x p}$ for the different leptons which in the SM are all equal (see for instance [38]). Thus, comparing tau decays to muons and tau decays to electrons we have (since in the SM $G_{a \rightarrow b} \propto g_{a} g_{b}$ )

$$
\left(\frac{g_{\mu}^{e x p}}{g_{e}^{e x p}}\right)^{2}=\left(\frac{G_{\tau \rightarrow \mu}}{G_{\tau \rightarrow e}}\right)^{2} \approx 1+\frac{\sqrt{2}}{G_{F} m_{h}^{2}}\left(\left|f_{\mu \tau}\right|^{2}-\left|f_{e \tau}\right|^{2}\right) .
$$

Similarly

$$
\begin{aligned}
& \left(\frac{g_{\tau}^{\text {exp }}}{g_{\mu}^{e x p}}\right)^{2}=\left(\frac{G_{\tau \rightarrow e}}{G_{\mu \rightarrow e}}\right)^{2} \approx 1+\frac{\sqrt{2}}{G_{F} m_{h}^{2}}\left(\left|f_{e \tau}\right|^{2}-\left|f_{e \mu}\right|^{2}\right), \\
& \left(\frac{g_{\tau}^{\text {exp }}}{g_{e}^{\text {exp }}}\right)^{2}=\left(\frac{G_{\tau \rightarrow \mu}}{G_{\mu \rightarrow e}}\right)^{2} \approx 1+\frac{\sqrt{2}}{G_{F} m_{h}^{2}}\left(\left|f_{\mu \tau}\right|^{2}-\left|f_{e \mu}\right|^{2}\right) .
\end{aligned}
$$

Universality constraints are summarized in table II, where measured values are translated into $90 \%$ CL limits.

- $\ell_{a}^{-} \rightarrow \ell_{b}^{-} \gamma$ : In the case of transition amplitudes $a \neq b$ the interesting observable is the decay rate. We find (for calculations including singly and doubly charged scalars see for instance [21, 39, 40])

$$
\mathrm{R}\left(\ell_{a}^{-} \rightarrow \ell_{b}^{-} \gamma\right) \equiv \frac{\Gamma\left(\ell_{a}^{-} \rightarrow \ell_{b}^{-} \gamma\right)}{\Gamma\left(\ell_{a}^{-} \rightarrow \ell_{b}^{-} \nu \bar{\nu}\right)} \approx \frac{\alpha}{48 \pi}\left(\left|\frac{\left(f^{\dagger} f\right)_{a b}}{G_{F} m_{h}^{2}}\right|^{2}+16\left|\frac{\left(g^{\dagger} g\right)_{a b}}{G_{F} m_{k}^{2}}\right|^{2}\right) .
$$




\begin{tabular}{ccc}
\hline SM Test & Experiment & Bound $(90 \% \mathrm{CL})$ \\
\hline lept./hadr. univ. $\sum_{q=d, s, b}\left|V_{u q}^{\text {exp }}\right|^{2}=0.9992 \pm 0.0011$ & $\left|f_{e \mu}\right|^{2}<0.015\left(m_{h} / \mathrm{TeV}\right)^{2}$ \\
$\mu / e$ universality & $g_{\mu}^{\text {exp }} / g_{e}^{e x p}=1.0001 \pm 0.0020$ & $\left.|| f_{\mu \tau}\right|^{2}-\left|f_{e \tau}\right|^{2} \mid<0.05\left(m_{h} / \mathrm{TeV}\right)^{2}$ \\
$\tau / \mu$ universality & $g_{\tau}^{\text {exp }} / g_{\mu}^{e x p}=1.0004 \pm 0.0022$ & $\left.|| f_{e \tau}\right|^{2}-\left|f_{e \mu}\right|^{2} \mid<0.06\left(m_{h} / \mathrm{TeV}\right)^{2}$ \\
$\tau / e$ universality & $g_{\tau}^{\exp } / g_{e}^{\exp }=1.0004 \pm 0.0023$ & $\left.|| f_{\mu \tau}\right|^{2}-\left|f_{e \mu}\right|^{2} \mid<0.06\left(m_{h} / \mathrm{TeV}\right)^{2}$ \\
\hline
\end{tabular}

Table II: Constraints from universality of charged currents.

The factor 16 in front of the doubly charged contribution does not usually appear in the literature [27] and deserves a comment: the Feynman rule for the $k e_{a} e_{b}$ vertex contains a factor 2 when $a \neq b$ because there are two identical terms in the Lagrangian, but also the vertex with $a=b$ contains a factor 2 because there are two identical Wick contractions in this kind of vertices. This factor of 2 for identical particles was missed in [27] which led the authors do define new coupling constants with different factors of 2 for diagonal and non-diagonal terms ${ }^{7}$. It is also important to remark that the singly and doubly charged scalar contributions do not interfere because they couple to fermions with different chirality. Again we have to remember that $\operatorname{BR}\left(\ell_{a}^{-} \rightarrow \ell_{b}^{-} \gamma\right)=$ $\mathrm{R}\left(\ell_{a}^{-} \rightarrow \ell_{b}^{-} \gamma\right) \operatorname{BR}\left(\ell_{a}^{-} \rightarrow \ell_{b}^{-} \nu \bar{\nu}\right)$

- $\mu-e$ conversion in nuclei: The new scalars of the model do not couple to quarks and, therefore, do not generate a four-fermion operator that could contribute at tree level to $\mu-e$ conversion. However, radiative corrections, in particular those related with the $\mu-e-\gamma$ vertex, will contribute to the process. It is also clear that those corrections are tightly related to the $\mu \rightarrow e \gamma$ decay discussed above but are not identical because the photon in $\mu-e$ conversion is not on the mass shell. In fact in ref. [41] if was shown that in models with doubly charged scalars there is a logarithmic enhancement, $\log \left(q^{2} / m_{k}\right)$, of the $\mu-e$ conversion amplitude with respect to the $\mu \rightarrow e \gamma$ amplitude. At present the best limits come from $\mu-e$ conversion on $T i, \sigma\left(\mu^{-} T i \rightarrow e^{-T i}\right) / \sigma\left(e^{-} T i \rightarrow\right.$ capture $)<$ $4.3 \times 10^{-12}$ [37] which, when translated into limits on the couplings, are slightly worse than present $\mu \rightarrow e \gamma$ constraints, but one has to keep in mind that if $\mu \rightarrow e \gamma$ is relevant and if $\mu-e$ conversion limits are improved in the future it will also be relevant.

\footnotetext{
${ }^{7}$ One can also see that those results cannot be right because physical amplitudes should transform correctly under the flavour redefinitions of couplings in eq. (7).
} 


\begin{tabular}{cc}
\hline Experiment & Bound $(90 \% \mathrm{CL})$ \\
\hline$\delta a_{e}=(12 \pm 10) \times 10^{-12}$ & $r\left(\left|f_{e \mu}\right|^{2}+\left|f_{e \tau}\right|^{2}\right)+4\left(\left|g_{e e}\right|^{2}+\left|g_{e \mu}\right|^{2}+\left|g_{e \tau}\right|^{2}\right)<5.5 \times 10^{3}\left(m_{k} / \mathrm{TeV}\right)^{2}$ \\
$\delta a_{\mu}=(21 \pm 10) \times 10^{-10}$ & $r\left(\left|f_{e \mu}\right|^{2}+\left|f_{\mu \tau}\right|^{2}\right)+4\left(\left|g_{e \mu}\right|^{2}+\left|g_{\mu \mu}\right|^{2}+\left|g_{\mu \tau}\right|^{2}\right)<7.9\left(m_{k} / \mathrm{TeV}\right)^{2}$ \\
$B R(\mu \rightarrow e \gamma)<1.2 \times 10^{-11} r^{2}\left|f_{e \tau}^{*} f_{\mu \tau}\right|^{2}+16\left|g_{e e}^{*} g_{e \mu}+g_{e \mu}^{*} g_{\mu \mu}+g_{e \tau}^{*} g_{\mu \tau}\right|^{2}<3.4 \times 10^{-5}\left(m_{k} / \mathrm{TeV}\right)^{4}$ \\
$B R(\tau \rightarrow e \gamma)<1.1 \times 10^{-7} r^{2}\left|f_{e \mu}^{*} f_{\mu \tau}\right|^{2}+16\left|g_{e e}^{*} g_{e \tau}+g_{e \mu}^{*} g_{\mu \tau}+g_{e \tau}^{*} g_{\tau \tau}\right|^{2}<1.7\left(m_{k} / \mathrm{TeV}\right)^{4}$ \\
$B R(\tau \rightarrow \mu \gamma)<4.5 \times 10^{-8} r^{2}\left|f_{e \mu}^{*} f_{e \tau}\right|^{2}+16\left|g_{e \mu}^{*} g_{e \tau}+g_{\mu \mu}^{*} g_{\mu \tau}+g_{\mu \tau}^{*} g_{\tau \tau}\right|^{2}<0.7\left(m_{k} / \mathrm{TeV}\right)^{4}$ \\
\hline
\end{tabular}

Table III: Constraints from lepton number violating photon interactions.

- $a=(g-2) / 2$ : For diagonal transitions the model gives additional contributions to the anomalous magnetic moments of the leptons which are very well measured in the case of the electron and the muon. We find that the additional contribution to the $a_{a}$ of lepton $\ell_{a}$, in this model, is

$$
\delta a_{a} \equiv a_{a}^{e x p}-a_{a}^{S M}=-\frac{m_{a}^{2}}{24 \pi^{2}}\left(\frac{\left(f^{\dagger} f\right)_{a a}}{m_{h}^{2}}+4 \frac{\left(g^{\dagger} g\right)_{a a}}{m_{k}^{2}}\right) .
$$

It is important to remark that we always find a negative contribution (this is in agreement with old and well tested calculations [42, 43] ). In the case of the muon $a_{\mu}$, recent analyses of experimental data and theoretical calculations in the Standard Model suggest that the experimental measurement is slightly larger than the SM prediction (for a review see [44]). Several authors have tried to explain this $1 \sigma$ to $3 \sigma$ effect in different extensions of the SM. In particular, in [45] the charged scalars of the Zee model were used to increase the $a_{\mu}$ of the SM. We find this is not possible and instead we will use the $g-2$ of the muon (and also of the electron) to constrain the parameters of the model. The relevant constraints coming from flavour lepton number changing photon interactions are summarized in table III. Experimental limits on branching ratios are already provided in the literature [37] at 90\% CL. Results for $g-2$ are usually given as measurements on $\delta a_{a}$. We will use [46] $\delta a_{e}=(12 \pm 10) \times 10^{-12}$ and $\delta a_{\mu}=(21 \pm 10) \times 10^{-10}$. Notice that in both cases the central value is positive, while the model gives a negative contribution. To place $90 \%$ CL bounds in this situation we use the Feldman and Cousins prescription [47] which, for the values above, gives $\left|\delta a_{e}\right|<6.1 \times 10^{-12}$ and $\left|\delta a_{\mu}\right|<3.8 \times 10^{-10}$.

Since lepton number is not conserved, another interesting low energy process that could arise in the model is neutrinoless double beta decay $(0 \nu 2 \beta)$. In this model, the singly and doubly 
charged scalars do not couple to hadrons, this means that the $(0 \nu 2 \beta)$ rate is dominated by the Majorana neutrino mass exchange and it is proportional to the $\left|\left(\mathcal{M}_{\nu}\right)_{e e}\right|^{2}$ matrix element, therefore it will be addressed in section [V] when we discuss in detail the neutrino mass matrix constraints.

\section{Perturbativity constraints}

Beside requiring that the model produce acceptable (a) neutrino masses and mixings (b) low energy predictions, we also have to address theoretical questions related to the validity of the predictions and the consistency of the model. Indeed to be able to perform any calculation in this model we have to assume that perturbation theory can be used. This imposes strong constraints on the relevant couplings of the model. The Yukawa couplings of the new scalars receive loop corrections like

$$
\delta f \sim \frac{f^{3}}{(4 \pi)^{2}}, \quad \delta g \sim \frac{g^{3}}{(4 \pi)^{2}} .
$$

If the corrections are going to be much smaller than the couplings, the couplings must satisfy $f, g \ll 4 \pi$. Similarly, the trilinear coupling among charged scalars proportional to the parameter $\mu$ induces loop corrections to the charged scalar masses like

$$
\delta m_{k}^{2}, \delta m_{h}^{2} \sim \frac{\mu^{2}}{(4 \pi)^{2}} .
$$

Requiring that the corrections are much smaller than the masses implies $\mu \ll 4 \pi m_{h}, 4 \pi m_{k}$. Since it is difficult to establish the exact values of the couplings for which the perturbativity of the theory breaks down, we will encode this type of constraints in the single parameter $\kappa$ and will require

$$
\left|f_{a b}\right|<\kappa, \quad\left|g_{a b}\right|<\kappa, \quad \mu<\kappa \min \left(m_{h}, m_{k}\right)
$$

For the purposes of illustration we will take $\kappa=1$ or $\kappa=5$, the value $\kappa=5$ being rather conservative (for instance, the strong coupling constant, $g_{s}$, is considered to become nonperturbative at scales of about $1 \mathrm{GeV}$, at those scales $\alpha_{s}(1 \mathrm{GeV}) \sim 0.5$ and $\left.g_{s}(1 \mathrm{GeV}) \sim 2.5\right)$.

The parameter $\mu$ is important in the generation of neutrino masses, therefore the constraint $\mu<\kappa \min \left(m_{h}, m_{k}\right)$ is important. On the other hand, as seen in tables II, II, III, if the scalar masses are relatively light (around $1 \mathrm{TeV}$ or less), low energy processes already 
provide interesting limits on the charged scalar Yukawa couplings. However, if the charged scalars are heavier, the experimental limits on the new Yukawa couplings are so mild that may allow Yukawa couplings large enough to compromise the perturbative validity of the theory. Then, the perturbativity constraints we just discussed will become relevant.

\section{THE MODEL AT THE LHC}

Extra scalar degrees of freedom arise in many scenarios extending the weak interactions beyond the SM. In our case, the scalar sector is enlarged by the addition of two charged scalars: $h$ and $k$, which could be produced at the LHC if their masses are low enough. In particular, as we will see below, the LHC will be very well suited for searching the doubly charged scalar, $k$. Studies for searching doubly-charged scalars at future colliders have been directed in the past [48, 49, 50, 51, 52]. In general this scalar is taken to be one component of a weak triplet. Such triplets are well motivated on theoretical grounds, specially when considering left-right symmetric models. Our work differs essentially in the gauge charges of the scalars. Both, $h$ and $k$, are charged weak singlets that do not acquire a VEV. This makes the phenomenology different. More model independent studies have been also considered in the literature [53, 54].

Concerning experimental bounds, LEP searched for these scalars. Their pair production $\left(e^{+} e^{-} \rightarrow \gamma^{*} Z^{*} \rightarrow k^{++} k^{--}\right)$implies the bound $m_{k}>100 \mathrm{GeV}$ [55, 56, 57]. Single production via $e^{+} e^{-} \rightarrow$ kee as well as contributions to Bhabha scattering have been also studied by LEP [57, 58], but in these cases the bounds depend on the (unknown) values of the Yukawa couplings. Tevatron has also been used to set bounds on this kind of scalars [59, 60, 61]. Depending on the details of the model (couplings, decay channels,...) the mass is again found to be roughly above $100 \mathrm{GeV}$.

\section{A. Collider phenomenology}

\section{Production}

The extra scalars can be pair produced via a Drell-Yan process, fig. 2, Although this production mechanism presents the drawback of having a potentially high threshold due to the creation of two scalars, it has the important advantage of being proportional to their 


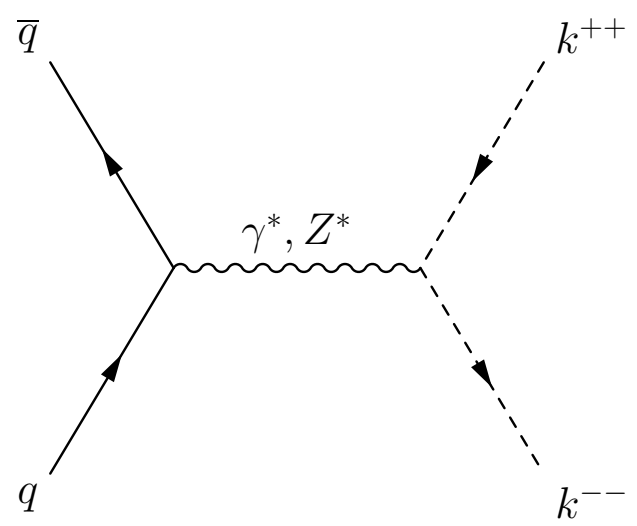

Figure 2: Pair production of $k$

gauge charges as well as depending only on one unknown parameter: the mass of the scalar. The partonic cross section at $\mathrm{LO}$ reads

$\sigma=\frac{\pi \alpha^{2} Q^{2} \beta^{3}}{6}\left[\frac{2 Q_{q}^{2}}{\hat{s}}-\frac{2\left(g_{L}+g_{R}\right) Q_{q}}{c_{w}^{2}} \frac{\hat{s}-M_{Z}^{2}}{\left(\hat{s}-M_{Z}^{2}\right)^{2}+\Gamma_{Z}^{2} M_{Z}^{2}}+\frac{\left(g_{L}^{2}+g_{R}^{2}\right)}{c_{w}^{4}} \frac{\hat{s}}{\left(\hat{s}-M_{Z}^{2}\right)^{2}+\Gamma_{Z}^{2} M_{Z}^{2}}\right]$,

where $\hat{s}$ is the energy squared in the center of mass frame (CM) of the quarks, $Q$ stands for electric charges, $g_{L}$ and $g_{R}$ are given for the quarks by $g_{L}=T_{3}-s_{w}^{2} Q_{q}$ and $g_{R}=-s_{w}^{2} Q_{q}$ and $\beta$ is the velocity of the produced scalars in this frame $\beta=\sqrt{1-4 m^{2} / \hat{s}}$.

Equation (30) shows that pair production is four times more efficient for $k$ than for $h$ due to their charges (assuming equal masses), which translates into a better discovery potential for $k$. The $k$ pair production cross section, $\sigma_{k k}$, at NLO for the LHC and Tevatron is displayed in fig. 3. To compute it, we have used CompHEP [62] with CTEQ6.1L libraries [63] to find the $\mathrm{LO}$ cross section and afterwards we have included a K-factor of 1.25 for the LHC and 1.3 for Tevatron to take into account NLO corrections, see [64].

Single production might be also interesting when double production is not possible. Single production can proceed with a $k$ accompanied by two singly charged scalars, fig. 4 , or by two charged leptons replacing the scalar $h$ 's. If the $k$ is accompanied by two charged leptons the amplitudes are proportional to the Yukawa couplings, whose exact values we ignore and might be small.

It is important to note that the cross section will be dominated by the virtual particles in the propagators if they could be on-shell. In the case of $k$ being produced with two $h$, the single production will be dominated by the first diagram if $\hat{s}>2 m_{k}$, because in this case $k^{*}$ can be created on-shell. One might argue that the energy in the center of mass frame of 


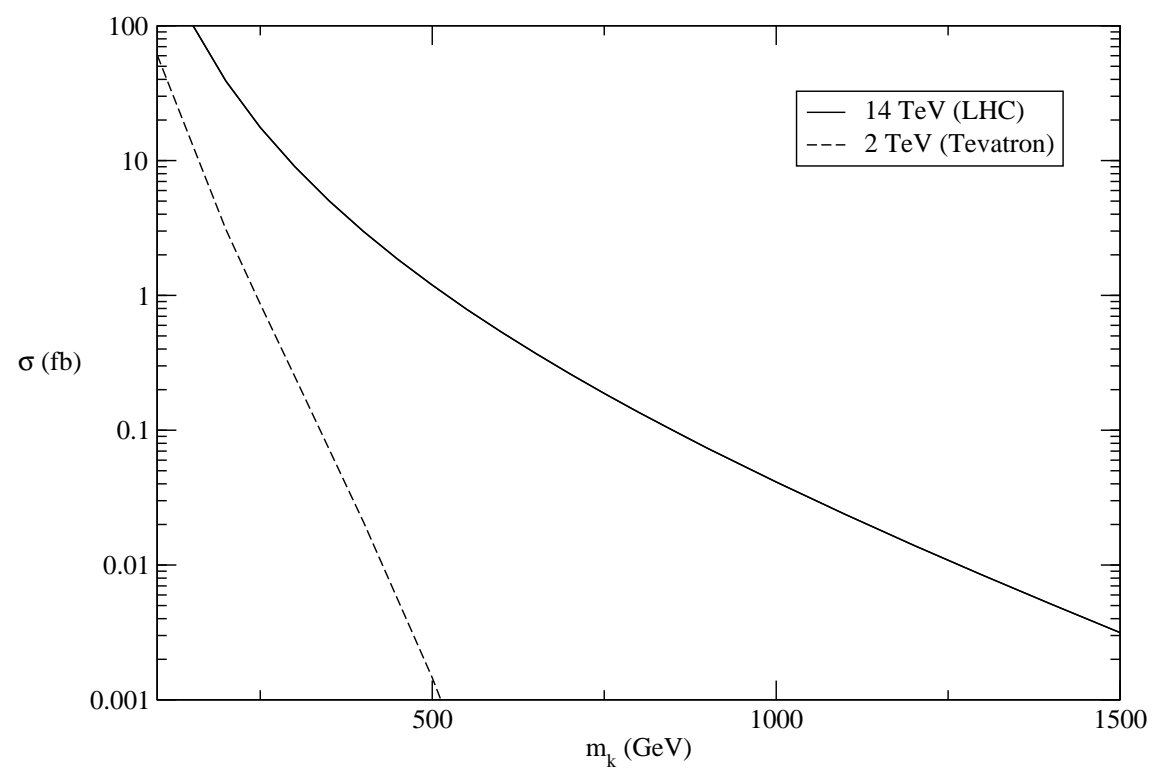

Figure 3: Pair production cross section for $k$. We have used CompHEP (CTEQ6.1L) to obtain the LO and applied a K-factor of 1.25 for the LHC and 1.3 for Tevatron.
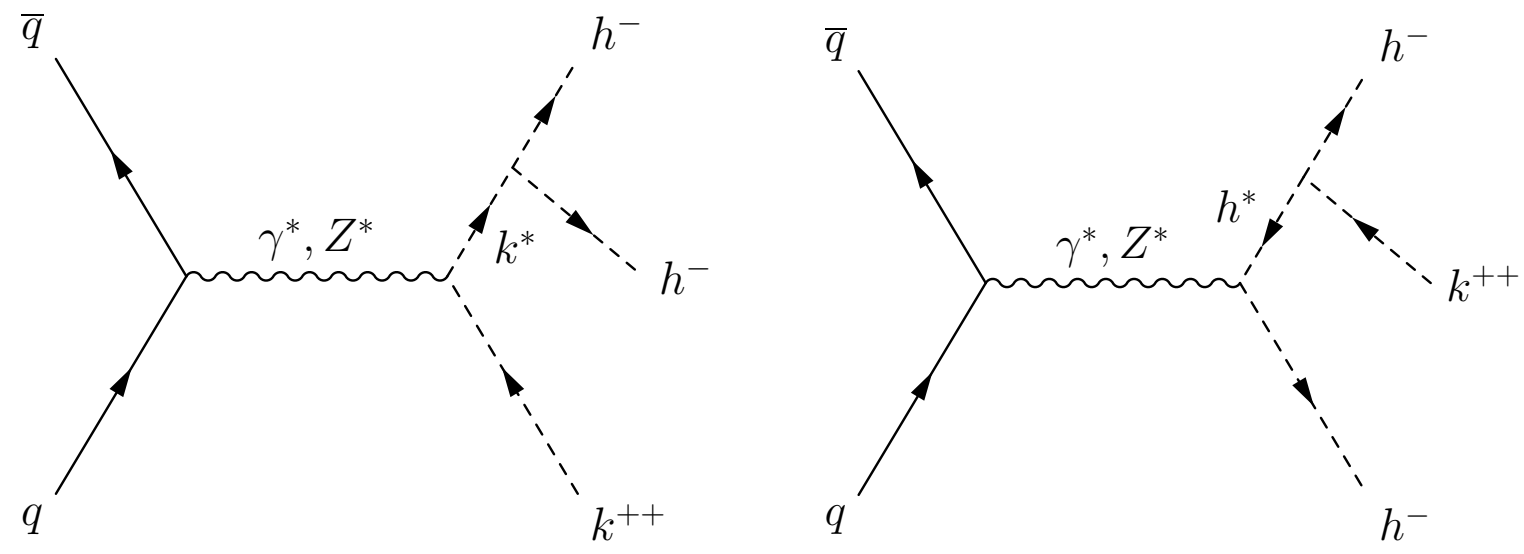

Figure 4: Single production diagrams.

the colliding quarks is not fixed, instead it is a fraction of the total energy in the center of mass frame of the colliding protons, $s$. However, the cross section involves an integration over the possible values of $\hat{s}$. If $s$ is large enough to create two $k$ 's, the integration will be dominated by the real production of two $k$ 's, thus reducing the single production to pair production. Specifically, $\sigma\left(k^{++} h^{-} h^{-}\right) \approx \sigma_{k k} \operatorname{Br}(k \rightarrow h h)$. The same reasoning is valid in the case of single production with leptons. We have performed calculations using CompHEP to check this point. Therefore, single production is only important when the available energy, $s$, is not sufficient to create a pair of $k$. 
A possible third production mechanism is via the couplings with the Higgs doublet, $H$. There is little information concerning these couplings because their contribution to low energy phenomenology is expected to be negligible in front of the Yukawas $f_{a b}$ and $g_{a b}$. This is so because the former enter at two loops and the later at tree level. In any case, the amplitudes of these processes are expected to be small because the Higgs couplings to quarks are proportional to their masses.

In summary, we find that, from the point of view of production, the best suited channel for discovery studies is pair production, being four times more efficient for $k$ than for $h$.

\section{Decay}

We assume for the moment that the scalars are not long lived, i.e. they decay before reaching the detector. Different decay channels present different experimental sensitivities depending on the final products. In particular, detectors are much less sensitive to those channels containing neutrinos and/or taus in the final states, since neutrinos (including those coming from the decay of the taus) will escape undetected. This makes necessary to compute the branching ratios.

The $k$ scalar can always decay to two leptons of the same sign, since $m_{k}>100 \mathrm{GeV}$. The width reads

$$
\Gamma\left(k \rightarrow \ell_{a} \ell_{b}\right)=\frac{\left|g_{a b}\right|^{2}}{4 \pi\left(1+\delta_{a b}\right)} m_{k}
$$

It is worth to stress that $k$ is the only particle in this model that can decay to two like-sign leptons, which will be crucial to detect it.

If $m_{k}>2 m_{h}$, then $k$ can also decay to a pair of $h$

$$
\Gamma(k \rightarrow h h)=\frac{1}{8 \pi}\left[\frac{\mu}{m_{k}}\right]^{2} m_{k} \sqrt{1-\frac{4 m_{h}^{2}}{m_{k}^{2}}} .
$$

On the other hand, the decay channels of $h$ reduce to those with one lepton and one neutrino in the final state:

$$
\Gamma\left(h \rightarrow \ell_{a} \nu_{b}\right)=\frac{\left|f_{a b}\right|^{2}}{4 \pi} m_{h}
$$

Since these channels involve always one neutrino it is clear that detecting $h$ will be much more complicated than detecting $k$ even if their production rates were similar. 
Finally we will check that the doubly charged scalar $k^{++}$cannot be long-lived in this model ${ }^{8}$ once low energy phenomenology and neutrino data are taken into account. Indeed the $k$ decay width can be written as

$$
\Gamma=\frac{m_{k}}{8 \pi}\left(\left|g_{\mu \mu}\right|^{2}+\cdots\right)
$$

where the $g_{\mu \mu}^{2}$ term takes into account the decay into muons and the dots represent all other possible couplings. Then, the long-lived condition translates into $\left|g_{\mu \mu}\right|<10^{-8}$ which cannot be fulfilled when the limits obtained in tabs. IV $\mathrm{V}$ are used.

\section{Detection at colliders}

As we have seen the discovery potential of the LHC for $k$ is more promising than for $h$. On one side because the production cross section of the former is enhanced with respect to the later and, on the other side, because the experimental sensitivity to the decay channels of $h$ is smaller. Thus, in the following we will focus on $k$.

After a pair of $k$ 's is created in the collider, they can decay into a number of final states. The most interesting for us contains four like-sign leptons. From now on we will refer to this channel as 4lep. The rest of the possible final states always contain $h$ or $\tau$. These channels are quite difficult to deal with experimentally because $\tau$ and $h$ will decay to neutrinos. In contrast, detection of electrons and muons is quite efficient. In addition, the decay of a $k$ to a pair of like-sign leptons $\left(e^{ \pm}\right.$or $\left.\mu^{ \pm}\right)$with high invariant masses constitutes a clear and distinct signature. This channel has a negligible background coming from SM processes, making it very appropriate for $k$ discovery studies.

In order to model the efficiencies and acceptances of the detectors at the LHC we use the criterion that 10 events of $k$ pair production with subsequent decay to 4lep lead to discovery of $k$. We expect that at least two of such events will be properly detected/identified providing us with four pairs of like-sign leptons which invariant mass will give us the first estimate of $m_{k}$. This criterion is taken from [50] where the authors perform a study of the discovery reach at Tevatron for doubly-charged bosons decaying to like-sign leptons in a similar model and a similar rule can be extracted from [52] where the authors focus on the ATLAS detector

\footnotetext{
${ }^{8}$ By "long-lived" we understand that the scalar can travel a distance of the order $c \tau>3 m[61]$.
} 


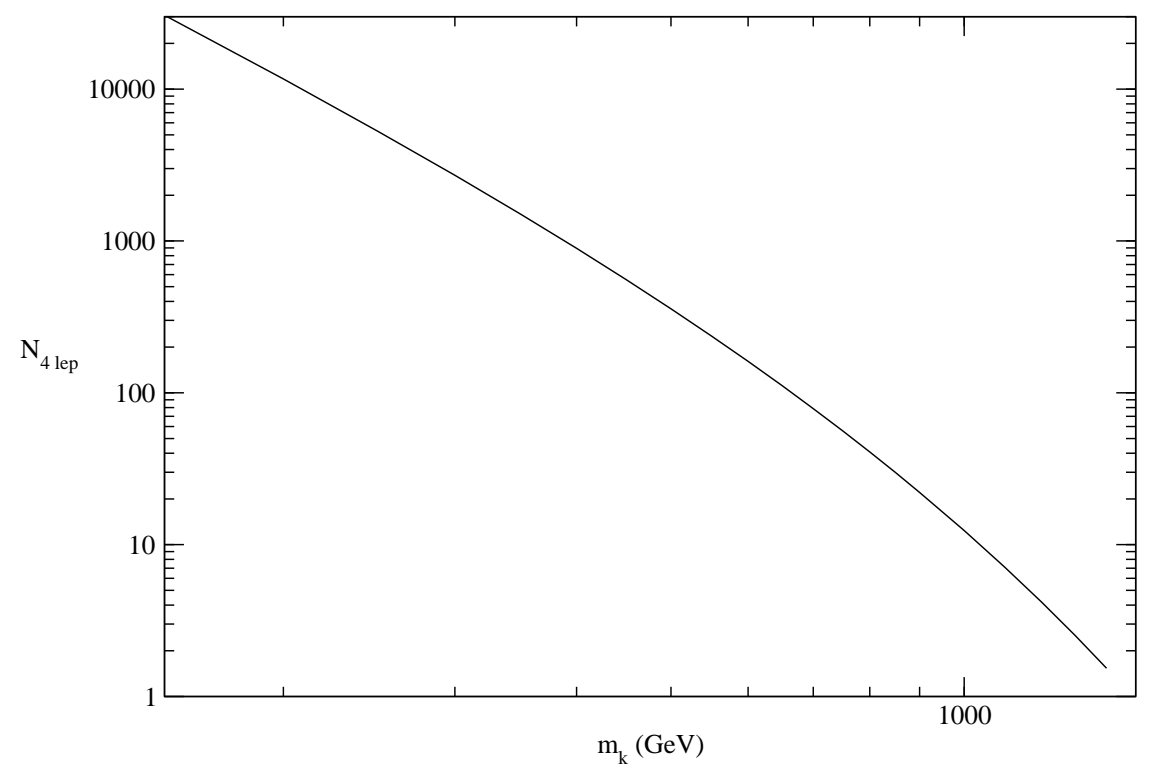

Figure 5: Number of events at the LHC in the 4lep channel for a luminosity $\mathcal{L}=300 \mathrm{fb}^{-1}$ and $\sqrt{s}=14 \mathrm{TeV}$ assuming that all produced $k$ pairs decay in this particular channel.

at the LHC. A more detailed study of the forthcoming detectors acceptances and efficiencies at the LHC is desirable.

To estimate the maximum reach in terms of $m_{k}$ at the LHC we take the most optimistic scenario in which all the $k$ pairs decay to 4lep. The number of events in this channel is shown in fig. 5 for the optimistic luminosity ${ }^{9} 300 \mathrm{fb}^{-1}$ and $\mathrm{CM}$ energy $14 \mathrm{TeV}$. From this plot one concludes that the LHC will be able to probe masses up to $1 \mathrm{TeV}$ approximately.

In general, the signal in the 4 lep channel will be smaller than the one shown in fig. 5 due to the presence of the other decay channels. This signal draining will be controlled by the branching ratio $B R_{4 l e p}$, which can be expressed in terms of the couplings as

$$
B R_{4 l e p}=\frac{\left|g_{e e}\right|^{2}+\left|g_{\mu \mu}\right|^{2}+2\left|g_{e \mu}\right|^{2}}{\left|g_{e e}\right|^{2}+\left|g_{\mu \mu}\right|^{2}+2\left|g_{e \mu}\right|^{2}+\left|g_{h h}\right|^{2}+2\left|g_{e \tau}\right|^{2}+2\left|g_{\mu \tau}\right|^{2}+\left|g_{\tau \tau}\right|^{2}}
$$

where we have defined the effective coupling of the doubly charged scalar to singly charged

\footnotetext{
9 The LHC luminosity is expected to be about $100 \mathrm{fb}^{-1} /$ year.
} 
scalars, $g_{h h}$, as

$$
g_{h h}=\left[\frac{\mu}{m_{k}}\right]\left(1-\frac{4 m_{h}^{2}}{m_{k}^{2}}\right)^{1 / 4} .
$$

\section{ANALYSIS OF THE PARAMETERS OF THE MODEL}

As discussed in section III the Yukawa couplings of the model can be written in terms of 12 moduli and 5 phases. Other parameters relevant for the model are the masses of the charged scalars, $m_{h}$ and $m_{k}$, and the coupling $\mu$. Among the 12 moduli, 3 correspond to the 3 charged lepton masses, which are known. Thus, we have 17 additional relevant parameters with respect to the plain SM with massless neutrinos (9 moduli and 5 phases from the new Yukawa couplings, the 3 scalar parameters, $m_{h}$ and $m_{k}$ and the coupling $\mu$ ). The remaining parameters in the scalar potential are of no interest for our purposes. The neutrino mass matrix is rather well known. In our case it contains 2 neutrino masses, 3 real mixing angles and 2 phases (1 CKM-type phase and 1 Majorana phase). Thus, there will still remain 4 moduli and 3 additional phases in the Yukawa couplings (plus $m_{h}, m_{k}$ and $\mu$ ). On most of these Yukawa couplings we have some information from section IIB as long as the masses of the charged scalars are not much heavier than $1 \mathrm{TeV}$. Notice that this is the interesting range for scalar masses if they are going to be produced at the LHC. In addition, there are also indirect arguments that suggest that the scalar masses should be relatively light (below $1 \mathrm{TeV}$ ) if one likes to avoid strong hierarchy problems ${ }^{10}$, since the charged scalar masses will contribute, at one loop, to the mass of the SM Higgs boson. However, the couplings of the SM Higgs boson to the new scalars are unknown and could be small. Thus, although the natural range of the masses of the new scalars is about few $\mathrm{TeV}$ or less, they can also be larger. Then, in what follows, we will allow the masses of the charged scalar to vary between the LEP lower bound $\sim 100 \mathrm{GeV}$ and infinity. We will immediately see, however, that present information already constrains the charged scalar masses to be below $\sim 10^{5} \mathrm{TeV}$. The couplings $g_{a b}, f_{a b}$ and $\mu$ must in addition satisfy the perturbativity constraints discussed in section ПC.

From the previous discussion it is clear that even though we have 17 additional parameters we also have a lot of information on them both from neutrino oscillations and from low energy

10 Alternatively one could enlarge the model by supersymmetrizing it. 
processes. However, the correlations among the different observables due to their dependence on the same set of parameters can be difficult to disentangle. Under those circumstances an adequate approach to the analysis of the Zee-Babu model should involve both an analytic understanding and a systematic numerical inspection of the parameter space to clarify the ranges allowed by the available experimental results for as many parameters and observables as possible.

We first exploit the information we have on neutrino oscillation experiments: the knowledge of two squared mass differences and the mixing angles.

With antisymmetric $f_{a b}$ 's, at two loops, the mass matrix determinant is equal to zero, and thus one eigenvalue is zero. Two mass differences are then sufficient to fix the masses both in the normal hierarchy and in the inverted hierarchy cases (a degenerate spectrum cannot arise in this model). Except for the Dirac phase $\delta$ and the Majorana phase $\phi$, we can almost reconstruct experimentally the neutrino mass matrix by using the information we have on the mixing angles and the masses. Without loss of generality we can write the neutrino Majorana mass matrix as

$$
\mathcal{M}_{\nu}=U D_{\nu} U^{T}
$$

with $U$ the standard PMNS matrix

$$
U=\left(\begin{array}{ccc}
1 & 0 & 0 \\
0 & c_{23} & s_{23} \\
0 & -s_{23} & c_{23}
\end{array}\right)\left(\begin{array}{ccc}
c_{13} & 0 & s_{13} e^{-i \delta} \\
0 & 1 & 0 \\
-s_{13} e^{i \delta} & 0 & c_{13}
\end{array}\right)\left(\begin{array}{ccc}
c_{12} & s_{12} & 0 \\
-s_{12} & c_{12} & 0 \\
0 & 0 & 1
\end{array}\right),
$$

while $D_{\nu}$ is the diagonal matrix of masses (including the only Majorana phase). Notice that writing the mass matrix in this form already implies some phase convention.

Since one of the $\nu$ masses of the model is zero we only have two possibilities ${ }^{11}$ :

Normal hierarchy $(\mathrm{NH})$

$$
D_{\nu}^{N H}=\left(\begin{array}{ccc}
0 & 0 & 0 \\
0 & m_{2} e^{i \phi} & 0 \\
0 & 0 & m_{3}
\end{array}\right), m_{3} \gg m_{2}, \begin{aligned}
& \Delta_{S}=m_{2}^{2} \\
& \Delta_{A}=m_{3}^{2}
\end{aligned}
$$

\footnotetext{
${ }^{11}$ Here we follow the conventions and results of ref. 65] adapted to our case.
} 
Inverted hierarchy (IH)

$$
D_{\nu}^{I H}=\left(\begin{array}{ccc}
m_{1} & 0 & 0 \\
0 & m_{2} e^{i \phi} & 0 \\
0 & 0 & 0
\end{array}\right), m_{1} \approx m_{2} \quad, \quad \begin{gathered}
\Delta_{S}=m_{2}^{2}-m_{1}^{2} \\
\Delta_{A}=m_{1}^{2}
\end{gathered}
$$

With

$$
\begin{gathered}
\Delta_{S}=(7.9 \pm 0.3) \times 10^{-5} \mathrm{eV}^{2} \\
\Delta_{A}=(2.5 \pm 0.25) \times 10^{-3} \mathrm{eV}^{2} \\
s_{12}^{2} \equiv \sin ^{2} \theta_{12}=0.30 \pm 0.03 \quad, \quad s_{13}^{2} \equiv \sin ^{2} \theta_{13} \leq 0.02, \quad 90 \% \mathrm{CL} . \\
s_{23}^{2} \equiv \sin ^{2} \theta_{23}=0.50 \pm 0.08
\end{gathered}
$$

Thus, apart from the poorly known $s_{13}$ mixing (we just know it is small) and the phases, $\delta$ and $\phi$, the mass matrix can be partially reconstructed in terms of the 2 known mass differences and the 2 known mixing angles for each of the two cases. In particular, we can immediately extract the matrix element responsible for $(0 \nu 2 \beta)$ decays:

Normal hierarchy

$$
\left\langle m_{\nu}^{N H}\right\rangle_{e e}=\left(\mathcal{M}_{\nu}^{N H}\right)_{e e}=\sqrt{\Delta_{S}} c_{13}^{2} s_{12}^{2} e^{i \phi}+\sqrt{\Delta_{A}} s_{13}^{2} e^{-i 2 \delta}
$$

In this case ${ }^{12}$, given the previous values, it is clear that $\left(\mathcal{M}_{\nu}^{N H}\right)_{e e} \lesssim 0.003 \mathrm{eV}$ and therefore difficult to see in $(0 \nu 2 \beta)$ decay experiments.

Inverted hierarchy

$$
\left\langle m_{\nu}^{I H}\right\rangle_{e e}=\left(\mathcal{M}_{\nu}^{I H}\right)_{e e}=\sqrt{\Delta_{A}+\Delta_{S}} c_{13}^{2} s_{12}^{2} e^{i \phi}+\sqrt{\Delta_{A}} c_{13}^{2} c_{12}^{2} .
$$

In this case, unless a cancellation occurs between the two terms for $e^{i \phi}=-1,\left(\mathcal{M}_{\nu}^{I H}\right)_{e e}$ is naturally of order $0.05 \mathrm{eV}$ and, therefore, observable in planned $(0 \nu 2 \beta)$ decay experiments.

Equation (13) gives the mass matrix $\mathcal{M}_{\nu}$ in terms of the parameters of the model - the Yukawa couplings, the scalar masses and the trilinear coupling -, we can thus try to fix some parameters by matching the $\mathcal{M}_{\nu}$, obtained from the neutrino oscillation parameters, to the calculated one. Since the mass matrix is symmetric, in principle this gives 6 equations. However, one of them is trivially satisfied because, by construction, both matrices already

12 Notice the dependence on the Dirac phase $\delta$. This is a consequence of our convention for Majorana phases. One could redefine phases and make this quantity independent on $\delta$, but this will not affect predictions or constraints on observables. 
satisfy $\operatorname{det}\left(\mathcal{M}_{\nu}\right)=0$. To choose the remaining 5 equations we will use that the eigenvector corresponding to the 0 eigenvalue is very simple; as $\operatorname{det} f=0$, there is an eigenvector a of $f$ with zero eigenvalue $f \cdot \mathbf{a}=0, \mathbf{a}=\left(f_{\mu \tau},-f_{e \tau}, f_{e \mu}\right)$. Obviously, a will also be an eigenvector of $\mathcal{M}_{\nu}$ with zero eigenvalue when expressed in terms of masses and mixings and, therefore, $U D_{\nu} U^{T} \mathbf{a}=0$ or, since $U$ is unitary, $D_{\nu} U^{T} \mathbf{a}=0$. This gives us three equations, one of which is satisfied trivially because one of the diagonal values of $D_{\nu}$ is zero. The other two equations will allow us to express the ratios of couplings $f_{i j}$ just in terms of mixing angles and phases ${ }^{13}$. Thus, in the NH case we have $\left(D_{\nu}^{N H}\right)_{11}=0$ and

$$
\begin{aligned}
& \left(U^{T} \mathbf{a}\right)_{2}=0 \Rightarrow \frac{f_{e \tau}}{f_{\mu \tau}}=\tan \theta_{12} \frac{\cos \theta_{23}}{\cos \theta_{13}}+\tan \theta_{13} \sin \theta_{23} e^{-i \delta} \\
& \left(U^{T} \mathbf{a}\right)_{3}=0 \Rightarrow \frac{f_{e \mu}}{f_{\mu \tau}}=\tan \theta_{12} \frac{\sin \theta_{23}}{\cos \theta_{13}}-\tan \theta_{13} \cos \theta_{23} e^{-i \delta}
\end{aligned}
$$

These equations immediately tell us that the standard PMNS convention of phases is not compatible with all $f_{a b}$ being real. However, we can take a phase convention in which $f_{\mu \tau}$ is real and positive and leave $f_{e \tau}$ and $f_{e \mu}$ complex with phases fixed by eq. (45).

With values like $s_{12}^{2} \sim 0.3, s_{23}^{2} \sim 0.5$ and $s_{13}^{2}<0.02$, the first term on the right hand side of eqs. (45) dominates and we get $f_{e \tau} \simeq f_{\mu \tau} / 2 \simeq f_{e \mu}$. With this relation we can go back to the low energy bounds in table II and table III and find that the strongest constraints on the $f_{i j}$ couplings come from $\mu \rightarrow e \gamma$ (which strongly bounds $\left|f_{e \tau} f_{\mu \tau}\right|$ ) and tell us that $\left|f_{e i}\right| \lesssim 0.05\left(m_{h} / \mathrm{TeV}\right)$ and $\left|f_{\mu \tau}\right| \lesssim 0.1\left(m_{h} / \mathrm{TeV}\right)$

The equations corresponding to the inverted hierarchy case, $\left(D_{\nu}^{I H}\right)_{33}=0$, are

$$
\begin{aligned}
& \left(U^{T} \mathbf{a}\right)_{1}=0 \Rightarrow \frac{f_{e \tau}}{f_{\mu \tau}}=-\frac{\sin \theta_{23}}{\tan \theta_{13}} e^{-i \delta} \\
& \left(U^{T} \mathbf{a}\right)_{2}=0 \Rightarrow \frac{f_{e \mu}}{f_{\mu \tau}}=\frac{\cos \theta_{23}}{\tan \theta_{13}} e^{-i \delta}
\end{aligned}
$$

In this case, it is clear that $f_{e \tau} / f_{e \mu}=-\tan \theta_{23} \approx-1$ and $\left|f_{e \mu}\right|>5\left|f_{\mu \tau}\right|,\left|f_{e \tau}\right|>5\left|f_{\mu \tau}\right|$. Now we can use these relations in the low energy bounds in table II and table III and find that the strongest constraints on the $f_{i j}$ couplings come from lepton-hadron universality (see table III), $5\left|f_{\mu \tau}\right| \lesssim\left|f_{e i}\right| \lesssim 0.1\left(m_{h} / \mathrm{TeV}\right)$.

We still have 3 additional equations we can use to fix the parameters of the model. In

13 Therefore, the decay branching ratios of the scalar $h$ to the different leptons are fixed by the mixing angles. This can probably be exploited [28] at the ILC . 
this case we have no good argument to choose them ${ }^{14}$ and following [27] we will take the three elements $m_{22}, m_{23}$ and $m_{33}$ in the equalities $m_{i j} \equiv\left(\mathcal{M}_{\nu}\right)_{i j}=\zeta f_{i a} m_{a} g_{a b}^{*} m_{b} f_{j b}$ with the $m_{i j}$ defined by eq. (37) and $\zeta=\frac{\mu}{48 \pi^{2} M^{2}} \tilde{I}$. Thus, if $\omega_{a b}=m_{a} g_{a b}^{*} m_{b}$ we have

$$
\begin{aligned}
& m_{22}=\zeta\left(f_{\mu \tau}^{2} \omega_{\tau \tau}-2 f_{e \mu} f_{\mu \tau} \omega_{e \tau}+f_{e \mu}^{2} \omega_{e e}\right) \\
& m_{23}=\zeta\left(-f_{\mu \tau}^{2} \omega_{\mu \tau}-f_{\mu \tau} f_{e \tau} \omega_{e \tau}+f_{\mu \tau} f_{e \mu} \omega_{e \mu}+f_{e \tau} f_{e \mu} \omega_{e e}\right) \\
& m_{33}=\zeta\left(f_{\mu \tau}^{2} \omega_{\mu \mu}+2 f_{\mu \tau} f_{e \tau} \omega_{e \mu}+f_{e \tau}^{2} \omega_{e e}\right) .
\end{aligned}
$$

Because of the hierarchy among the charged lepton masses, it is natural to assume that those $\omega_{a b}$ containing the electron mass, $\omega_{e e}, \omega_{e \mu}, \omega_{e \tau}$, are much smaller than $\omega_{\mu \mu}, \omega_{\mu \tau}, \omega_{\tau \tau}$, in that case we can neglect them (we will check later the goodness of this approximation within the numerical analysis), and we have

$$
m_{22} \simeq \zeta f_{\mu \tau}^{2} \omega_{\tau \tau}, m_{23} \simeq-\zeta f_{\mu \tau}^{2} \omega_{\mu \tau}, m_{33} \simeq \zeta f_{\mu \tau}^{2} \omega_{\mu \mu}
$$

In the normal hierarchy case this gives $\left(s_{i j} \equiv \sin \theta_{i j}, c_{i j} \equiv \cos \theta_{i j}\right)$

$$
\begin{aligned}
& \zeta f_{\mu \tau}^{2} \omega_{\tau \tau} \simeq m_{3} c_{13}^{2} s_{23}^{2}+m_{2} e^{i \phi}\left(c_{12} c_{23}-e^{i \delta} s_{12} s_{13} s_{23}\right)^{2} \\
& \zeta f_{\mu \tau}^{2} \omega_{\mu \tau} \simeq-m_{3} c_{13}^{2} c_{23} s_{23}+m_{2} e^{i \phi}\left(c_{12} s_{23}+e^{i \delta} c_{23} s_{12} s_{13}\right)\left(c_{12} c_{23}-e^{i \delta} s_{12} s_{13} s_{23}\right), \\
& \zeta f_{\mu \tau}^{2} \omega_{\mu \mu} \simeq m_{3} c_{13}^{2} c_{23}^{2}+m_{2} e^{i \phi}\left(c_{12} s_{23}+e^{i \delta} c_{23} s_{12} s_{13}\right)^{2} .
\end{aligned}
$$

With $m_{3} \simeq 0.05 \mathrm{eV}$ and $m_{2} \simeq 0.009 \mathrm{eV}$,

$$
\left|\omega_{\tau \tau}\right| \simeq\left|\omega_{\mu \tau}\right| \simeq\left|\omega_{\mu \mu}\right| \simeq \frac{0.05 \mathrm{eV}}{2 \zeta\left|f_{\mu \tau}\right|^{2}},
$$

setting a definite hierarchy among the $g_{a b}$ couplings:

$$
g_{\tau \tau}: g_{\mu \tau}: g_{\mu \mu} \sim m_{\mu}^{2} / m_{\tau}^{2}: m_{\mu} / m_{\tau}: 1
$$

In the inverted hierarchy case, eqs. (47) give

$$
\begin{aligned}
\zeta f_{\mu \tau}^{2} \omega_{\tau \tau} & \simeq m_{1}\left(c_{23} s_{12}+e^{i \delta} c_{12} s_{13} s_{23}\right)^{2}+m_{2} e^{i \phi}\left(c_{12} c_{23}-e^{i \delta} s_{12} s_{13} s_{23}\right)^{2} \\
\zeta f_{\mu \tau}^{2} \omega_{\mu \tau} & \simeq m_{1}\left(s_{12} s_{23}-e^{i \delta} c_{12} c_{23} s_{13}\right)\left(c_{23} s_{12}+e^{i \delta} c_{12} s_{13} s_{23}\right) \\
& +m_{2} e^{i \phi}\left(c_{12} s_{23}+e^{i \delta} c_{23} s_{12} s_{13}\right)\left(c_{12} c_{23}-e^{i \delta} s_{12} s_{13} s_{23}\right) \\
\zeta f_{\mu \tau}^{2} \omega_{\mu \mu} & \simeq m_{1}\left(s_{12} s_{23}-e^{i \delta} c_{12} c_{23} s_{13}\right)^{2}+m_{2} e^{i \phi}\left(c_{12} s_{23}+e^{i \delta} c_{23} s_{12} s_{13}\right)^{2}
\end{aligned}
$$

\footnotetext{
${ }^{14}$ Except that they cannot be in the same column or the same row of $\mathcal{M}_{\nu}$, because in that case the equations are related by $\mathcal{M}_{\nu} \mathbf{a}=0$.
} 
where $m_{1} \simeq m_{2} \simeq 0.05 \mathrm{eV}$, also yielding for $e^{i \phi}=1$

$$
\left|\omega_{\tau \tau}\right| \simeq\left|\omega_{\mu \tau}\right| \simeq\left|\omega_{\mu \mu}\right| \simeq \frac{0.05 \mathrm{eV}}{2 \zeta\left|f_{\mu \tau}\right|^{2}}
$$

and the hierarchy of couplings in eq. (51). However, in the IH case there is a strong cancellation for Majorana phases close to $\pi$, and one can obtain a smaller value for $\omega_{\mu \mu}$, thus we can only write

$$
\left|\omega_{\mu \mu}\right|>\frac{0.007 \mathrm{eV}}{2 \zeta\left|f_{\mu \tau}\right|^{2}} .
$$

In both cases one expects $g_{\mu \mu}$ to be the largest coupling among the three considered. Of course, $g_{e e}, g_{e \mu}$ and $g_{e \tau}$ can also be large and are only constrained by low energy processes and perturbativity constraints. One should notice, however, that in the inverted hierarchy case, the approximation made in going from eqs. (47) to eqs. (48) may be a priori less justifiable than in the normal hierarchy case when $\theta_{13} \rightarrow 0$, as the eigenvector corresponding to the zero eigenvalue, $\left(f_{\mu \tau},-f_{e \tau}, f_{e \mu}\right)$ is proportional to $\left(e^{i \delta} \tan \theta_{13}, \sin \theta_{23}, \cos \theta_{23}\right)$, i.e. $f_{\mu \tau} \propto \tan \theta_{13}$,

and since the terms retained in eq. (47) are proportional to $f_{\mu \tau}$, it is not obvious that the terms proportional to $\omega_{e i}$ can be neglected.

Assuming then that $\left|g_{\mu \tau}\right| \approx\left|g_{\mu \mu}\right|\left(m_{\mu} / m_{\tau}\right)$ and $\left|g_{\tau \tau}\right| \approx\left|g_{\mu \mu}\right|\left(m_{\mu} / m_{\tau}\right)^{2}$, we can go back to tables \and III to find the relevant constraints on the couplings. The best constraint comes from $\tau^{-} \rightarrow \mu^{+} \mu^{-} \mu^{-}$, which tells us that $\left|g_{\mu \mu}\right| \lesssim 0.4\left(m_{k} / \mathrm{TeV}\right),\left|g_{\mu \tau}\right| \lesssim 0.024\left(m_{k} / \mathrm{TeV}\right)$, $\left|g_{\tau \tau}\right| \lesssim 0.0015\left(m_{k} / \mathrm{TeV}\right)$.

We can use all this information to set analytical bounds on the relevant parameters of the model in the line discussed at the beginning of section $\amalg \mathrm{A}$.

\section{A. Analytical constraints}

1. NH case

First, just from the neutrino mass formula, we have

$$
\left|g_{\mu \mu}\right|\left|f_{\mu \tau}\right|^{2} \geq 10^{-3} \frac{\max \left(m_{k}, m_{h}\right)}{\tilde{I} \mathrm{TeV}} \frac{\max \left(m_{k}, m_{h}\right)}{\mu} .
$$

Now we can show that due to the logarithmic growth of $\tilde{I}$ for $m_{k} \gg m_{h}$ and the fact that $\tilde{I} \leq 1$ for $m_{k}<m_{h}$, 


$$
\frac{\max \left(m_{k}, m_{h}\right)}{\widetilde{I} m_{h}} \geq 1
$$

Thus

$$
\left|g_{\mu \mu}\right|\left|f_{\mu \tau}\right|^{2} \geq 10^{-3} \frac{m_{h}}{\mathrm{TeV}} \frac{\max \left(m_{k}, m_{h}\right)}{\mu} .
$$

Now we use the perturbativity bound on $\mu, \mu<\kappa \min \left(m_{h}, m_{k}\right)$

$$
\kappa\left|g_{\mu \mu}\right|\left|f_{\mu \tau}\right|^{2} \geq 10^{-3} \frac{m_{h}}{\mathrm{TeV}} \frac{\max \left(m_{k}, m_{h}\right)}{\min \left(m_{k}, m_{h}\right)}
$$

which can be rewritten as (use that $m_{h} m_{k}=\max \left(m_{k}, m_{h}\right) \min \left(m_{k}, m_{h}\right)$ )

$$
\left(\frac{\max \left(m_{k}, m_{h}\right)}{\mathrm{TeV}}\right)^{2} \leq 10^{3} \kappa\left|g_{\mu \mu}\right|\left|f_{\mu \tau}\right|^{2} \frac{m_{k}}{\mathrm{TeV}}
$$

We can use that $m_{k} \leq \max \left(m_{k}, m_{h}\right)$ and the perturbative constraints $\left|g_{\mu \mu}\right|<\kappa,\left|f_{\mu \tau}\right|<\kappa$ to find an upper limit on the masses of the charged scalars

$$
m_{h}, m_{k} \leq \max \left(m_{k}, m_{h}\right)<10^{3} \kappa^{4} \mathrm{TeV}
$$

On the other hand, if we use $\left|g_{\mu \mu}\right| \lesssim 0.4\left(m_{k} / \mathrm{TeV}\right)$, coming from $\tau \rightarrow 3 \mu$ and $\left|f_{\mu \tau}\right| \lesssim$ $0.1\left(m_{h} / \mathrm{TeV}\right)$, coming from $\mu \rightarrow e \gamma$ we immediately obtain a lower bound on the masses of the scalars

$$
m_{k}, m_{h}>\min \left(m_{k}, m_{h}\right)>\frac{0.51}{\sqrt{\kappa}} \mathrm{TeV}
$$

If we only use the $\tau \rightarrow 3 \mu$ constraint in (58) we find a bound on the $\left|f_{\mu \tau}\right|$ coupling

$$
\kappa\left|f_{\mu \tau}\right|^{2} \geq 2.6 \times 10^{-3}\left(\frac{m_{h}}{\min \left(m_{k}, m_{h}\right)}\right)^{2},
$$

and using that $m_{h}>\min \left(m_{k}, m_{h}\right)$ we find an absolute limit on the coupling

$$
\left|f_{\mu \tau}\right|>\frac{0.051}{\sqrt{\kappa}} .
$$

Thus, using either the experimental bounds and/or the perturbativity bounds, we can also set upper and lower limits on the different couplings $\left|g_{\mu \mu}\right|, \mu$ and the interesting observables $B R(\mu \rightarrow e \gamma)$ and $B R(\tau \rightarrow 3 \mu)$. As discussed in section III the LHC will be able to find the doubly charged scalar of the model $k^{++}$as long as it is lighter than about $1 \mathrm{TeV}$, thus it is interesting to know what are the constraints on the parameters of the model if $m_{k}<1 \mathrm{TeV}$. Following the procedure described above one can also put strong limits on the parameters of 


\begin{tabular}{|c|c|}
\hline General case & $m_{k}<1 \mathrm{TeV}$ \\
\hline \hline$\frac{0.51}{\sqrt{\kappa}} \mathrm{TeV} \leq m_{h}, m_{k}<10^{3} \kappa^{4} \mathrm{TeV}$ & $\frac{0.51}{\sqrt{\kappa}} \mathrm{TeV} \leq m_{h}, m_{k}<24 \kappa^{3 / 2} \mathrm{TeV}$ \\
\hline$\frac{0.1}{\kappa} \mathrm{TeV}<\mu<10^{3} \kappa^{5} \mathrm{TeV}$ & $0.26 \mathrm{TeV}<\mu<\kappa \mathrm{TeV}$ \\
\hline$\frac{0.051}{\sqrt{\kappa}}<\left|f_{\mu \tau}\right|<\kappa, \frac{0.01}{\kappa^{2}} \leq\left|g_{\mu \mu}\right| \leq \kappa$ & $\frac{0.051}{\sqrt{\kappa}}<\left|f_{\mu \tau}\right|<\kappa, \frac{0.1}{\kappa} \leq\left|g_{\mu \mu}\right| \leq \kappa$ \\
\hline$B R(\mu \rightarrow e \gamma) \geq 1.0 \times 10^{-19} / \kappa^{12}$ & $B R(\mu \rightarrow e \gamma) \geq 8 \times 10^{-13} / \kappa^{2}$ \\
\hline$B R(\tau \rightarrow 3 \mu) \geq 1.5 \times 10^{-18} / \kappa^{12}$ & $B R(\tau \rightarrow 3 \mu) \geq 2 \times 10^{-10} / \kappa^{4}$ \\
\hline
\end{tabular}

Table IV: Normal Hierarchy analytical constraints: we assume $\omega_{i e} \simeq 0$ and central values for measured oscillation parameters. For other experimental information we use 90\% CL. The dependence on the perturbativity constraints is encoded in the parameter $\kappa$ and explicitly displayed.

the model adding this additional constraint. We collect all the limits we obtain in table IV] It is important to remark the assumptions we use to obtain these bounds: we assume that because the small electron mass, as compared with the tau lepton and muon masses, $\omega_{i e} \simeq 0$. We also take central values for the measured oscillation parameters. For other experimental information, bounds on branching ratios of rare processes, we use $90 \%$ CL limits. Finally the dependence on the perturbativity constraints is encoded in the parameter $\kappa$ and explicitly displayed.

\section{IH case}

The same kind of bounds can be obtained for the IH case with a few remarks. In the IH case $f_{\mu \tau}$ is not the largest coupling among the $f^{\prime} s$, since $\left|f_{\mu \tau}\right| \approx \sqrt{2} s_{13}\left|f_{e \mu}\right|$ with $s_{13}$ small. Thus perturbativity bounds should be applied to $f_{e \mu}$. In addition the best experimental limit is also on $f_{e \mu},\left|f_{e \mu}\right|<0.1\left(m_{h} / \mathrm{TeV}\right)$. Then, it is convenient to write the main equations in terms of $f_{e \mu}$ instead of $f_{\mu \tau}$. Finally in the IH hierarchy case there is the possibility of 


\begin{tabular}{|c|c|}
\hline General case & $m_{k}<1 \mathrm{TeV}$ \\
\hline$\frac{0.95}{\sqrt{\kappa}} \mathrm{TeV}<m_{k}, m_{h}<274 \kappa^{4} \mathrm{TeV}$ & $\frac{0.95}{\sqrt{\kappa}} \mathrm{TeV} \leq m_{h}, m_{k}<11 \kappa^{3 / 2} \mathrm{TeV}$ \\
\hline$\frac{0.36}{\kappa} \mathrm{TeV}<\mu<274 \kappa^{5} \mathrm{TeV}$ & $0.9 \mathrm{TeV}<\mu<\kappa \mathrm{TeV}$ \\
\hline$\frac{0.095}{\sqrt{\kappa}}<\left|f_{e \mu}\right|<\kappa, \frac{0.036}{\kappa^{2}} \leq\left|g_{\mu \mu}\right| \leq \kappa$ & $\frac{0.1}{\sqrt{\kappa}}<\left|f_{e \mu}\right|<\kappa, \frac{0.36}{\kappa} \leq\left|g_{\mu \mu}\right| \leq \kappa$ \\
\hline$B R(\mu \rightarrow e \gamma) \geq 2 \times 10^{-18} / \kappa^{12}$ & $B R(\mu \rightarrow e \gamma) \geq 1 \times 10^{-12} / \kappa^{2}$ \\
\hline$B R(\tau \rightarrow 3 \mu) \geq 2 \times 10^{-16} / \kappa^{12}$ & $B R(\tau \rightarrow 3 \mu) \geq 3 \times 10^{-8} / \kappa^{4}$ \\
\hline $0.0007 / \kappa^{3}<s_{13}^{2}<0.02$ & $0.018 / \kappa<s_{13}^{2}<0.02$ \\
\hline
\end{tabular}

Table V: Inverse Hierarchy analytical constraints.

cancellations for $\phi=\pi$ which allow for a slightly smaller $\omega_{\mu \mu}$. We have in this case

$$
s_{13}^{2}\left|g_{\mu \mu}\right|\left|f_{e \mu}\right|^{2} \geq 7.3 \times 10^{-5} \frac{m_{h}}{\mathrm{TeV}} \frac{\max \left(m_{k}, m_{h}\right)}{\mu} .
$$

Then we can repeat essentially the same arguments used for the NH, together with the upper limit on $s_{13}^{2}, s_{13}^{2}<0.02$, to obtain lower and upper limits on the masses of the scalars, $m_{h}, m_{k}$, on the coupling $\left|f_{e \mu}\right|$, which is related to $\left|f_{e \tau}\right|$ and $\left|f_{\mu \tau}\right|$, on the coupling $\left|g_{\mu \mu}\right|$, related to $\left|g_{\mu \tau}\right|$ and $\left|g_{\tau \tau}\right|$, and on the trilinear coupling $\mu$. In addition, since in the IH case there is a strong dependence on $s_{13}^{2}$ we can also set a lower bound on it. As in the NH case we also give the corresponding limits one would find under the assumption that the double charged scalar $k^{++}$is found at the LHC and, therefore, has a mass smaller than $1 \mathrm{TeV}$. We summarize all the limits in table $\mathrm{V}$.

\section{B. Numerical analysis}

The information we obtained above is very useful; however, to obtain it we have made use of different approximations: 
a) We assumed that the $\omega_{e e}, \omega_{e \mu}, \omega_{e \tau}$ can be neglected in front of the other couplings. This approximation is reasonable because these $\omega$ 's are proportional to the electron mass, $\omega_{e i}=m_{e} g_{e i} m_{i}$, which is much smaller than the other two lepton masses. However, it could happen that, for some reason, the $g_{e i}$ couplings are much larger than the others. It is therefore important to perform a complete analysis without this assumption.

b) We took central values for the measured oscillation parameters.

c) In the analytical limits we only used data from neutrino oscillations and bounds from $\tau \rightarrow 3 \mu$ and $\mu \rightarrow$ er (or lepton/hadron universality in the IH case) together with the perturbativity constraints. As discussed in section IIB there are many more experimental constraints that can affect the results and should be taken into account.

It is clear that the only way to analyze the model without those approximations is by means of an exhaustive numerical exploration of the parameter space of the model. The basic tool to achieve this goal will be the use of Monte Carlo (MC) techniques; however, because of the large number of independent parameters and their diverse relevance, straightforward application of MC techniques is not sufficiently efficient and thus some additional considerations and refinements will be required.

The crudest MC exploration of the available parameter space would involve random generation of complete sets of 17 independent basic parameters, calculation of the corresponding predictions for the observables and finally an acceptation/rejection process in terms of the agreement between those predictions and the appropriate experimental constraints. Beside the large number of parameters to be considered, the relations among them previously discussed render such a crude approach almost hopeless.

Realizing that not all observables play an equal role, that is, some of them are much more informative or constraining than others, we can go one further step in the use of simple MC techniques: instead of the simplest MC outlined above, we can construct a MC process devised to automatically produce mass matrices in agreement with neutrino oscillation experiments.

Knowing the masses and mixing angles, if we were to reconstruct the mass matrix $\mathcal{M}_{\nu}$ using experimental input, the only missing ingredients would be the Dirac phase $\delta$, the Majorana phase $\phi$ (see eqs. (38 40) ) and the poorly known mixing $\theta_{13}$, for which we only know it is small and ignore its exact value or even if it is zero. Equation (13) gives the mass 
matrix $\mathcal{M}_{\nu}$ in terms of the new parameters - the Yukawa couplings, the scalar masses and the trilinear coupling -, we can thus try to fix some parameters by matching the extracted $\mathcal{M}_{\nu}$ from oscillation data and the calculated one. This procedure achieves two goals: it guarantees that neutrino oscillations are adequately produced and it reduces the freedom in parameter space entering numerical study by trading some of the couplings by measured neutrino oscillation parameters. For each set $\left\{\theta_{12}, \theta_{13}, \theta_{23}, \Delta_{A}, \Delta_{S}, \delta, \phi\right\}$ we thus obtain numerical values for the entries in $\mathcal{M}_{\nu}$. We will then use eqs. (45) or eqs. (46) to fix $f_{e \mu}$ and $f_{e \tau}$ in terms of $f_{\mu \tau}$ and the generated mixing angles. Then come eqs. (47); these three complex relations involve the six complex couplings $g_{a b}$, the trilinear coupling and the scalar masses. Together with $m_{k}, m_{h}$ and $\mu$, knowing three independent $g_{a b}$ 's in eqs. (47) will be sufficient to fix the remaining ones; effectively this means that we will generate $g_{e e}, g_{e \mu}$ and $g_{e \tau}$, and thus automatically fix $g_{\mu \mu}, g_{\mu \tau}$ and $g_{\tau \tau}$. Notice that this phase convention is compatible with the standard choice for the neutrino mass matrix, eqs. (37, 38).

To summarize, by generating five quantities $-\Delta_{A}, \Delta_{S}, \theta_{i j}$ - according to experimental knowledge, two phases $-\delta$ and $\phi-$, one real coupling $f_{\mu \tau}$, two masses $-m_{h}$ and $m_{k}-$, the trilinear coupling $\mu$ and three complex $g_{a b}$, we are spanning the 12 moduli and 5 phases needed to describe the model. That is, instead of the crude and utterly inefficient Monte Carlo procedure in terms of $\left\{m_{k}, m_{h}, \mu, f_{a b}, g_{a b}\right\}$, we can use $\left\{s_{i j}^{2}, \Delta_{A}, \Delta_{S}, \delta, \phi, f_{\mu \tau}, m_{h}, m_{k}, \mu, g_{e e}, g_{e \mu} g_{e \tau}\right\}$ to explore the whole parameter space and guarantee the agreement with neutrino oscillations results prior to the use of the remaining experimental constraints, which constitute the next step, as they are then applied to accept/reject "candidate points". Notice that we have not specified the generation process of the different quantities involved: some discussion will be addressed below, the details of the numerical generation are summarized in table VI.

Despite being operative and useful, this refined MC procedure is not the last word as one can do better. For this purpose we resort to the use of Markov Chain driven Monte Carlo (MCMC) processes of the Metropolis type.

We have discussed the benefits of a refined simple MC procedure with respect to the crudest one: the next (and final) step to complete the numerical toolkit we use is the rather straightforward conversion of this refined MC into a Metropolis-like simulation which provides the results to be discussed. This is largely beneficial as (1) the efficiency of the MCMC process is sufficient to produce a reliable and smooth output for the different subcases under study, (2) the refined MC gives a helpful check of the consistency of the whole process. 


\begin{tabular}{|c|c|c||c|c|c|}
\hline Parameter & Value & Shape & Parameter & Value & Shape \\
\hline$\Delta_{S}$ & $(7.9 \pm 0.3) \times 10^{-5} \mathrm{eV}^{2}$ & Flat & $\Delta_{A}$ & $(2.5 \pm 0.25) \times 10^{-3} \mathrm{eV}^{2}$ & Flat \\
\hline $\sin ^{2} \theta_{12}$ & $0.30 \pm 0.03$ & Flat & $\sin ^{2} \theta_{23}$ & $0.50 \pm 0.08$ & Flat \\
\hline $\sin ^{2} \theta_{13}$ & {$\left[10^{-7} ; 2 \times 10^{-2}\right]$} & Log flat & & & \\
\hline \hline$\delta$ & {$[0 ; 2 \pi[$} & Flat & $\phi$ & {$[0 ; 2 \pi[$} & Flat \\
\hline$m_{h}$ & {$\left[10^{2} ; 10^{9}\right] \mathrm{GeV}$} & Log Flat & $m_{k}$ & {$\left[10^{2} ; 10^{9}\right] \mathrm{GeV}$} & Log Flat \\
\hline$f_{\mu \tau}$ & {$\left[10^{-7} ; \kappa\right]$} & Log flat & $\mu$ & {$\left[1 ; 10^{10}\right] \mathrm{GeV}$} & Log flat \\
\hline$\left|g_{e e}\right|$ & {$\left[10^{-7} ; \kappa\right]$} & Log flat & $\arg \left(g_{e e}\right)$ & {$[0 ; 2 \pi[$} & Flat \\
\hline$\left|g_{e \mu}\right|$ & {$\left[10^{-7} ; \kappa\right]$} & Log flat & $\arg \left(g_{e \mu}\right)$ & {$[0 ; 2 \pi[$} & Flat \\
\hline$\left|g_{e \tau}\right|$ & {$\left[10^{-7} ; \kappa\right]$} & Log flat & $\arg \left(g_{e \tau}\right)$ & {$[0 ; 2 \pi[$} & Flat \\
\hline
\end{tabular}

Table VI: Numerical values.

Let us now discuss the remaining details concerning the simulations; notice that, even if in the following we refer to the generation of parameters, which is appropriate for the MC process, the corresponding feature when dealing with MCMC processes is not generation but in fact how they enter the stepwise acceptance function, however, to avoid essentially duplicated discussions we will just mention what concerns the plain MC case. The main idea that drives our election of shapes and ranges of the different parameters is the need to perform an adequate exploration of the available parameter space, in particular one has to ensure that the regions which can yield interesting signals like the production of scalars at the LHC or branching ratios of exotic processes close to present experimental bounds are properly studied. In particular:

- Neutrino oscillations results, i.e. the squared masses differences $\Delta_{A}, \Delta_{S}$ and the mixing parameters $\sin ^{2} \theta_{12}, \sin ^{2} \theta_{23}$, are generated with flat distributions within a $\pm 1.64 \sigma$ range around the quoted experimental value (this corresponds to $90 \%$ confidence level or probability region for a gaussian-distributed uncertainty of the measurement). For $\sin ^{2} \theta_{13}$, however, we only have an upper bound: to span a reasonable range of values it is generated through a logarithmically flat distribution from the upper bound down to very small values, cut off at $10^{-7}$.

- The Dirac and Majorana phases, $\delta$ and $\phi$, are generated according to flat distributions 
spanning the whole available range $[0 ; 2 \pi[$.

- Concerning the independent Yukawa couplings $f_{a b}$ and $g_{a b}$, moduli are generated through distributions logarithmically flat to explore values that could potentially span several orders of magnitude. The applied upper bounds correspond to the different naturalness/perturbative cases under consideration. The arguments, as the Dirac and Majorana phases, are generated through flat distributions over the complete $[0 ; 2 \pi[$ range.

- The masses of the new scalar fields $m_{k}$ and $m_{h}$ are generated with logarithmically flat distributions reaching up to $10^{5} \mathrm{TeV}$ and bounded below at $\sim 100 \mathrm{GeV}$ to incorporate LEP-motivated constraints. In any case, the precise upper bound is irrelevant as far as it is beyond the analytic bounds presented in tables [V] and V]

- The trilinear coupling $\mu$ is also generated with a distribution flat in its logarithm and limited by the perturbativity requirement.

- We apply the remaining experimental constraints presented in section IB in a sharp (straightforward acceptance or rejection) way: the only acceptable predictions are the ones within the quoted $90 \%$ CL ranges/bounds.

- The simulation described in the previous points allows a very wide range of scalar masses. However, as discussed, the most interesting case is when $m_{k}<1 \mathrm{TeV}$ and, therefore, the $k^{++}$can be discovered at the LHC. Thus, we have performed an independent simulation requiring $m_{k}<1 \mathrm{TeV}$.

- All the simulations are done for both the $\mathrm{NH}$ and $\mathrm{IH}$ cases and for two values of the perturbativity constraint $\kappa=1$ and $\kappa=5$.

The arbitrariness in the choice of priors and their impact in the final results is always a concern in this type of analyses. Because of this we have used several priors. In the case of the neutrino oscillation parameters we have repeated the analysis fixing the parameters at the central values, taking gaussian distributions around central values and using the flat distributions we have finally presented here. The differences are marginal and we chose to present results for flat distributions because the results are slightly more conservative. For other parameters we also tried plain flat priors, but, specially for parameters that range 
in several orders of magnitude, logarithmically flat distributions span more efficiently the parameter space. We checked that the distributions obtained for the observables considered, for which we found analytical lower and upper limits, do not depend too much on the choice.

At this point the machinery used to perform the announced numerical studies has been completely presented, however some comments on the nature and interpretation of the output it produces are in order.

The model under study naturally "lives" in a parametric space of high dimensionality. The standard statistical arsenal offers two different approaches to reduce this high dimensional information and produce tolerably low dimensional - usually one or two dimensional - output: the frequentist and the bayesian frameworks. Very schematically:

- Frequentists assign confidence levels to the marginalized output through the best fit achievable with the remaining parametric freedom.

- Bayesians assign probability densities to the marginalized output through the integration over the remaining parametric freedom of the likelihood (of data for the given parameters) times the prior distribution/weight of parameters (this is just Bayes conditional probability inversion formula at work).

Beside the long standing quarrel existing among practitioners of one or the other approach, both, with their information reduction schemes, unavoidably present some drawbacks together with their statistical merits. As we want sensitivity to the parameter space available for the model to work, the procedure we have followed might look quite bayesian. Being aware of the dependence on prior election and the imprecise nature of the details behind many constraints ${ }^{15}$, we do not intend at all to try and produce would-be highly orthodox statistical results neither interpret them as if they were so, and thus we have chosen the numerical details of the simulations - that is both experimental constraints and priors - as stated above without any further qualm.

\footnotetext{
${ }^{15}$ Usually the available information is just a $90 \%$ CL range and little additional knowledge on the distribution originating this range is given. Moreover the perturbativity constraints, as clearly seen in tables [V] and $\mathrm{V}$ are determinant and, like all theoretical constraints, no obvious confidence levels or statistical significance can be assigned to them.
} 


\section{RESULTS}

In this section we collect the main results of the paper.

First we would like to study the impact of the different assumptions and experimental data in the analysis.

To illustrate the impact of low energy constraints $(\mu \rightarrow e \gamma, \tau \rightarrow 3 \mu, \ldots)$ we perform an independent simulation with only neutrino data and another simulation including all constraints (in the case of $\mathrm{IH}$ and $\kappa=5$ ) and represent the resulting distribution ${ }^{16}$ of $m_{k}$ for the two simulations. We represent with a dashed line the results of the simulation only with neutrino data and with a solid line the simulation with all present experiments included.

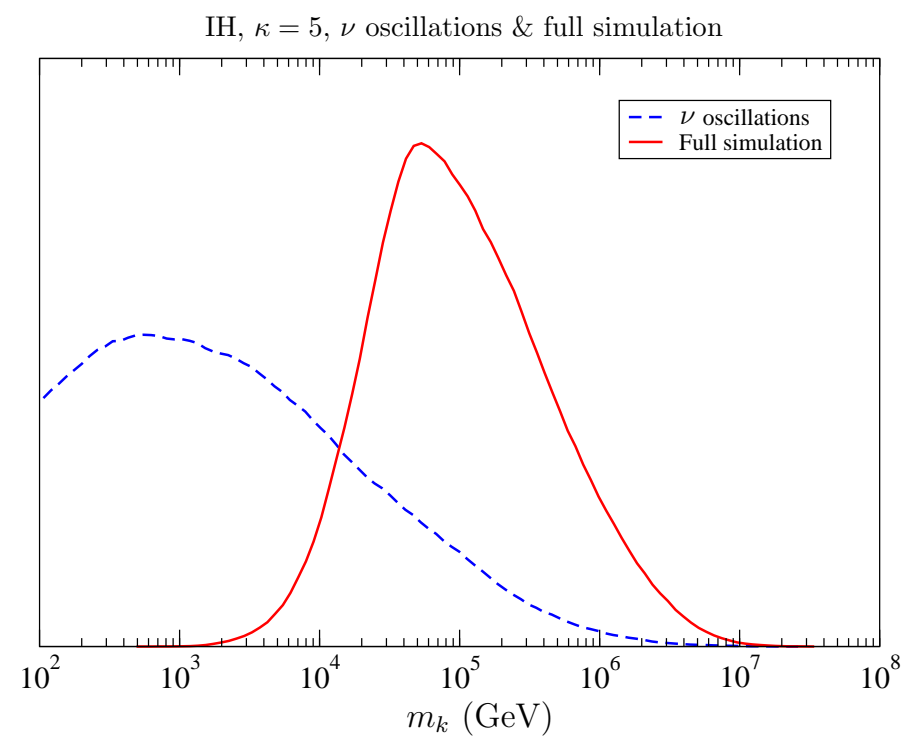

Figure 6: Impact of low energy constraints $(\mu \rightarrow e \gamma, \tau \rightarrow 3 \mu, \ldots): m_{k}$ distribution when only neutrino data is included (dashed) as compared with the case in which all experiments are included (solid). Displayed data correspond to the IH case and $\kappa=5$.

It is clear from the figure that only neutrino data allow (even prefer) relatively low masses of the order of $1 \mathrm{TeV}$ or below. However, when low energy experimental data is included the lower limit on the $m_{k}$ is pushed to larger values. We have to remark that the shape of the curves basically reflects the volume of the parameter space (from the other parameters)

${ }^{16}$ In the following we obtain the distributions as five million point samples from a MCMC exploration of the parameter space as described in section IVB. 
and that the tails can be rather long, thus regions below $m_{k}<1 \mathrm{TeV}$, as we will see by performing simulations with $m_{k}<1 \mathrm{TeV}$, are not completely forbidden.

Similarly, to illustrate the impact of the perturbativity constraint, we represent in fig. 7 the $m_{h}$ distribution from a simulation with $\kappa=1$ and another one with $\kappa=5$ (both in IH case with all experimental information included).

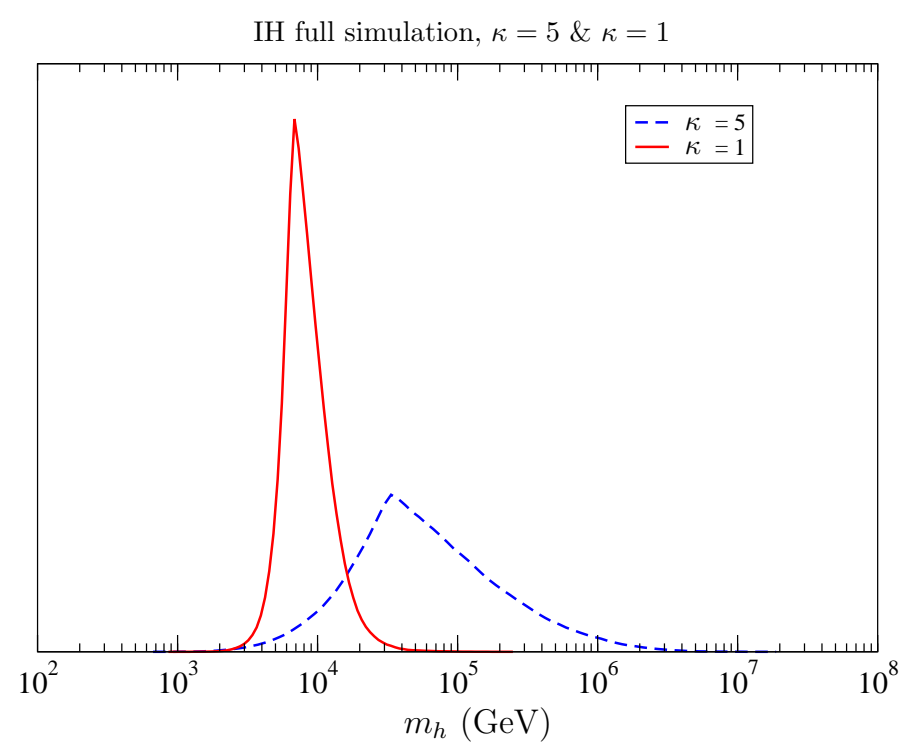

Figure 7: Impact of the perturbativity constraints: $m_{h}$ distribution for $\kappa=1$ (dashed) and $\kappa=5$ (solid). IH case with all experimental data included.

We confirm with this figure the scaling of the bounds with the perturbativity assumptions, encoded in the parameter $\kappa$, obtained analytically. Thus, for smaller values of $\kappa$ the allowed range of $m_{h}$ is much smaller. For $\kappa=1$, the preferred region of $m_{h}$ is in the range $\sim$ $1-100 \mathrm{TeV}$ (although with long tails) while for $\kappa=5$ this range is enlarged to $1-10000 \mathrm{TeV}$. Although it cannot be appreciated in this figure the lower bound on the mass is also sensitive to $\kappa$ as shown analytically (see table $\mathrm{V}$ ).

From figure 6 it is clear that all present data allow a wide range of $k^{++}$masses however, the $k^{++}$, as discussed in III, can only be discovered at the LHC if $m_{k}<1 \mathrm{TeV}$. Thus, this is the really interesting region of parameters to be studied. To study this region we perform an independent simulation implementing (we present results for the IH case and $\kappa=5$ ) all present constraints but assuming, in addition, that the $k^{++}$has been discovered at the LHC and therefore has a mass $m_{k}<1 \mathrm{TeV}$. In fig. 8 we present the $B R(\mu \rightarrow e \gamma)$ distribution in the two cases, general case and $m_{k}<1 \mathrm{TeV}$. We see the dramatic impact in $B R(\mu \rightarrow e \gamma)$ 
of the discovery of the $k^{++}$at the LHC. While present data allow branching ratios in the range $10^{-22}-10^{-11}$, if the $k^{++}$is discovered at the LHC then $B R(\mu \rightarrow e \gamma) \sim 10^{-13}-10^{-11}$ and, therefore, will be probed at the MEG experiment.

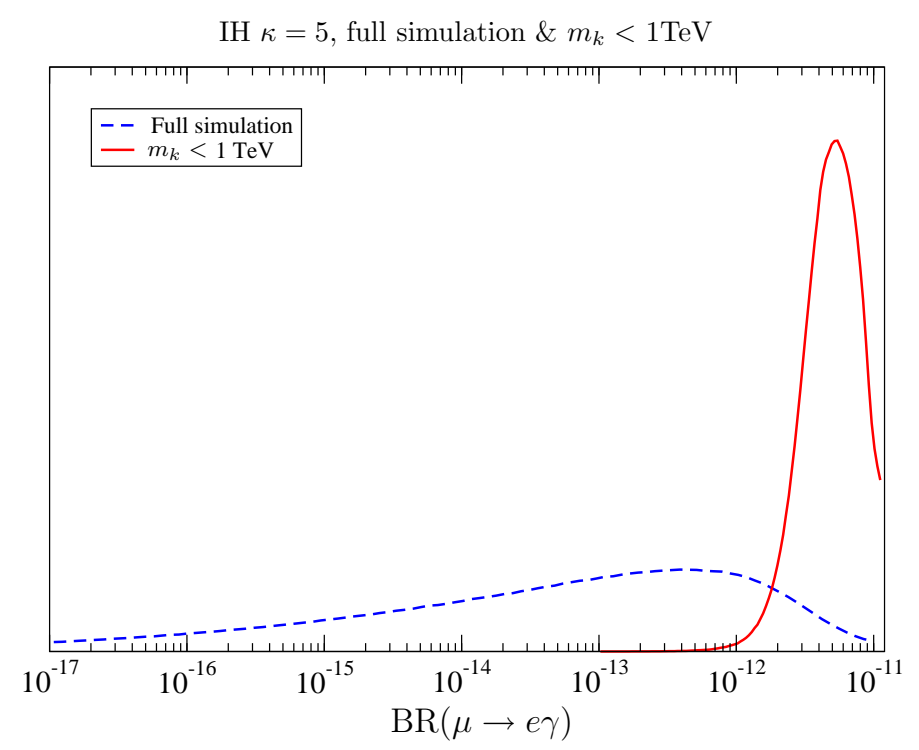

Figure 8: Impact of the discovery of the $k^{++}$at the LHC $\left(m_{k}<1 \mathrm{TeV}\right): B R(\mu \rightarrow e \gamma)$ distribution for the general case, IH and $\kappa=5$ with all present experimental results, (dashed) and requiring in addition that $k^{++}$has been seen at the $\mathrm{LHC}\left(m_{k}<1 \mathrm{TeV}\right) \kappa=5$ (solid).

Until now we have presented results only for the IH case. In general, as also seen in our approximate analytical results, we expect roughly similar results in the NH and the IH case, except for a few parameters and/or observables. In particular we mentioned that in the IH case there is a preference for the Majorana phase around $\phi=\pi$ because in that case there is a cancellation in the neutrino mass formulas. This is confirmed by the numerical calculation: in fig. 9 we represent the distribution of $\phi$ for both, the NH case (dashed line) and the IH case (solid line). The data is taken from a simulation with $\kappa=5$, including all present experimental constraints and requiring that $m_{k}<1 \mathrm{TeV}$. The distribution in the $\mathrm{NH}$ case is practically flat, while in the IH case it is highly peaked at $\phi=\pi$.

We also expect large differences in the $\mathrm{NH}$ and $\mathrm{IH}$ cases for the parameter $\sin ^{2} \theta_{13}$. In fig. 10 we represent the $\sin ^{2} \theta_{13}$ distribution for the two cases, $\mathrm{NH}$ and $\mathrm{IH}(\kappa=5$, full data and $\left.m_{k}<1 \mathrm{TeV}\right)$. While it is constant in the NH case, in the IH case it is highly peaked at the maximum values allowed by present data and, in fact, there is an absolute lower bound on it, $\sin ^{2} \theta_{13} \gtrsim 2 \times 10^{-3}$, which is not so far from the present upper limit, $\sin ^{2} \theta_{13}<2 \times 10^{-2}$. 


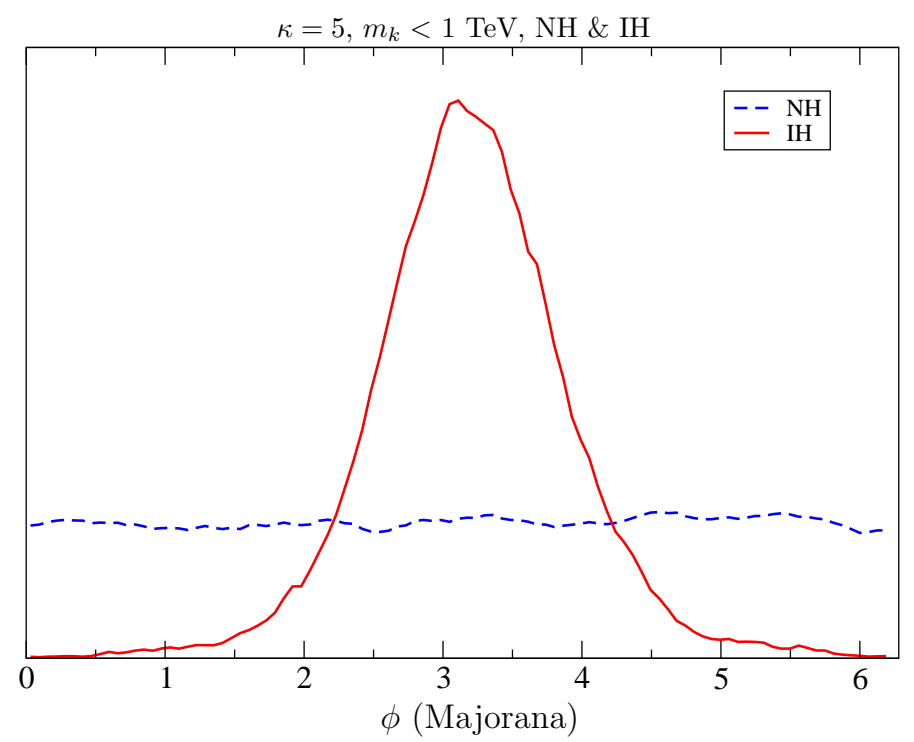

Figure 9: Differences between the NH and IH cases: distribution of the Majorana phase $\phi$ : dashed in the $\mathrm{NH}$ case and solid in the $\mathrm{IH}$ case. All present experimental data included and $\kappa=5$.

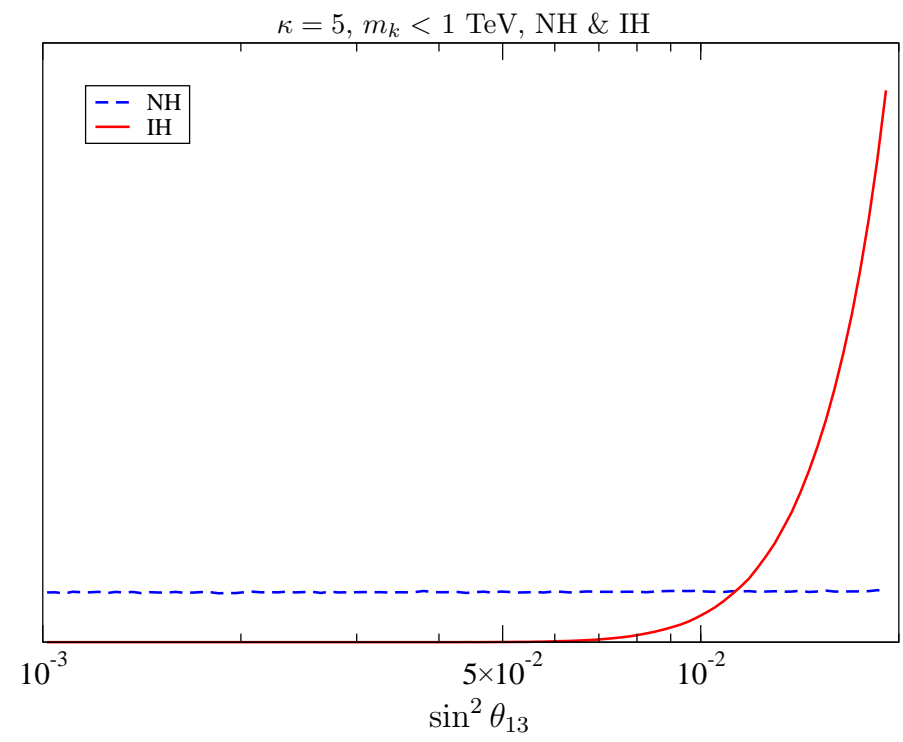

Figure 10: Differences between the NH and IH cases: distribution of $\sin ^{2} \theta_{13}$ as in fig. 9,

Finally, to illustrate another interesting difference between the two cases, NH and IH, we have represented in fig. 11 the distribution of $\left\langle m_{\nu}\right\rangle_{e e}$, the relevant matrix element in the neutrinoless double beta decay experiments. As before we assume $\kappa=5$ and $m_{k}<1 \mathrm{TeV}$, however, this assumption has little influence on the result since, as shown in eqs. 43. 44), $\left\langle m_{\nu}\right\rangle_{e e}$ is a function of only the neutrino masses and the mixing angles. Thus, the shape of the curves and their position is just a consequence of the fact that the model 
predicts a massless neutrino. In any case, from the figure it is clear that the model predicts $\left\langle m_{\nu}\right\rangle_{e e} \sim 0.001-0.005 \mathrm{eV}$ in the $\mathrm{NH}$ and $\left\langle m_{\nu}\right\rangle_{e e} \sim 0.01-0.06 \mathrm{eV}$ in the IH case.

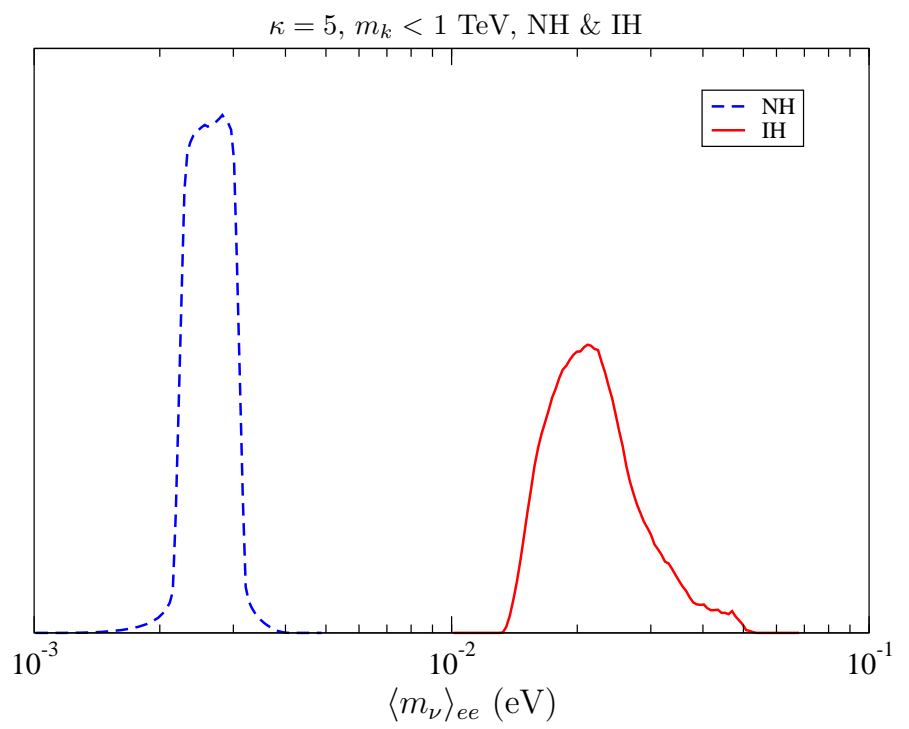

Figure 11: Predictions for $\left\langle m_{\nu}\right\rangle_{e e}$ in the NH (dashed) and the IH (solid) cases. All present experimental data included and $\kappa=5$.

Now, for the most interesting observables, those which give some interesting constraints or good perspectives in future tests, we present two-dimensional contour plots of the corresponding distributions. The density of points has been calculated using 50 bins in a logarithmic scale for each axis. Then, 10 contour lines equally spaced, ranging from the maximum density to 1/1000 of it, have been represented. Thus, the last contour region, painted with a lighter color, represents the region with a small density of points but which still contains some points. For each pair of observables we present two plots. On the left we present the distribution when all present experimental constraints are imposed. The values in the interesting region for the LHC $\left(m_{k}<1 \mathrm{TeV}\right)$ are very low but not zero. Thus, to better study this region we present in a second plot (right) the results of a simulation imposing the additional constraint $m_{k}<1 \mathrm{TeV}$. All results are given for the most conservative perturbativity assumption $(\kappa=5)$. Scaling for more restrictive assumptions can be inferred from tables IV and $\mathrm{IV}$. We discuss the relevant plots for both the $\mathrm{NH}$ and $\mathrm{IH}$ cases. 


\section{A. Normal Hierarchy}

Here we consider correlations among observables in the normal hierarchy case.
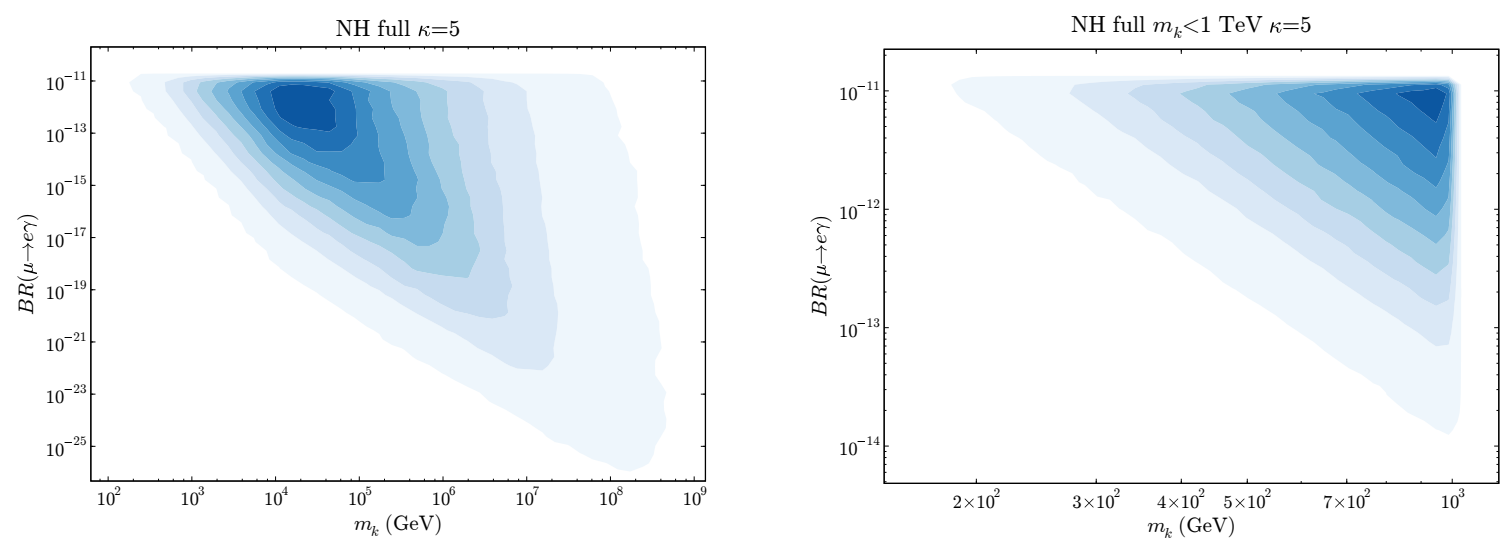

Figure 12: NH: $B R(\mu \rightarrow e \gamma)$ vs $m_{k}$; (left) general case (right) assuming that the $k^{++}$is seen at the LHC $\left(m_{k}<1 \mathrm{TeV}\right)$.

The most interesting observables of the model are $m_{k}$, because, if small enough, it will be accessible at the LHC, and the $B R(\mu \rightarrow e \gamma)$, which will be probed at a precision of the order of $10^{-13}$ in the MEG experiment. Thus in fig. 12 we display the joint $B R(\mu \rightarrow e \gamma)-m_{k}$ distribution. We observe a clear correlation between these two observables. Present data (left) seems to prefer $m_{k} \sim 10 \mathrm{TeV}$ and $B R(\mu \rightarrow e \gamma)$ above $10^{-13}$, but a large region of values is not excluded $m_{k} \sim 10^{2}-10^{8} \mathrm{GeV}$ and $B R(\mu \rightarrow e \gamma) \sim 10^{-25}-10^{-11}$, however if the doubly charged scalar, $k^{++}$, is discovered at the LHC $\left(m_{k}<1 \mathrm{TeV}\right)$ the situation changes dramatically and the simulation shows that the preferred values are in the upper range $^{17} m_{k} \gtrsim 600 \mathrm{GeV}$ and $B R(\mu \rightarrow e \gamma) \gtrsim 10^{-12}$ and that $k^{++}$masses below $200 \mathrm{GeV}$ and $B R(\mu \rightarrow e \gamma)$ below $10^{-14}$ are very difficult to obtain in the model.

Since $B R(\mu \rightarrow e \gamma)$ depends more explicitly on $m_{h}$ than on $m_{k}$ it is interesting to study the correlation between $B R(\mu \rightarrow e \gamma)$ and $m_{h}$. In fig. 13 we depict the allowed region in the plane $B R(\mu \rightarrow e \gamma)-m_{h}$, on the left for the general case and on the right for the case $m_{k}<1 \mathrm{TeV}$. We see a strong correlation specially in the general case. From the figure on the left we see that in the general case $m_{h}$ can be in a very wide range of values $m_{h} \sim 10^{2}-10^{8} \mathrm{GeV}$ but the preferred values are $m_{h} \sim 40 \mathrm{TeV}$. On the other hand, if $m_{k}<1 \mathrm{TeV}$ the allowed range

${ }^{17}$ For specific numbers we take 3 contours in the plots. 
of $m_{h}$ is much smaller, $m_{h} \sim 10^{2}-10^{5} \mathrm{GeV}$, and is shifted to the lower edge. It still allows a large range of masses not accessible at the LHC.
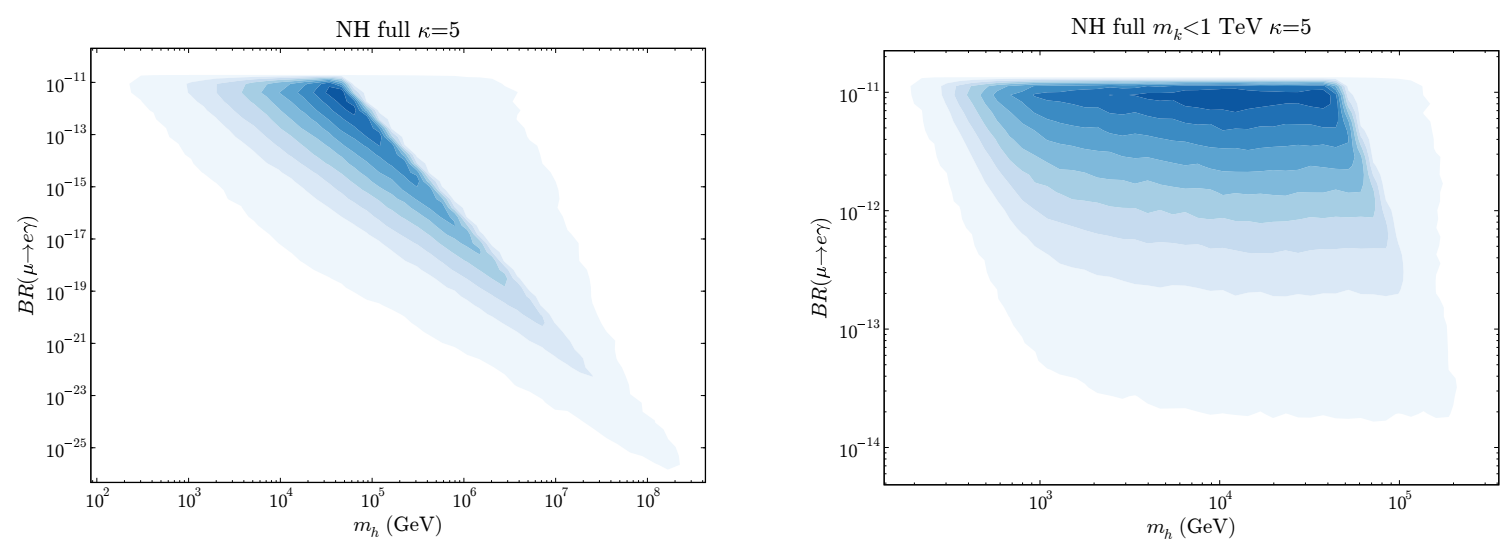

Figure 13: NH: $B R(\mu \rightarrow e \gamma)$ vs $m_{h}$.

$\tau \rightarrow 3 \mu$ is mediated by $k^{++}$exchange and governed by couplings which are largely fixed by neutrino mass data. Thus in fig. 14 we present results for the allowed values in the plane $B R(\tau \rightarrow 3 \mu)-m_{k}$. As expected there is a strong correlation in the two cases considered. In the general case the preferred values of $B R(\tau \rightarrow 3 \mu)$ are in the $10^{-13}$ range, although values as small as $10^{-25}$ are allowed. In the $m_{k}<1 \mathrm{TeV}$ case the preferred values are $B R(\tau \rightarrow 3 \mu) \gtrsim 10^{-9}$ which is not so far from present limits, $B R(\tau \rightarrow 3 \mu)<3.2 \times 10^{-8}$, but values like $10^{-13}$ are not completely excluded.
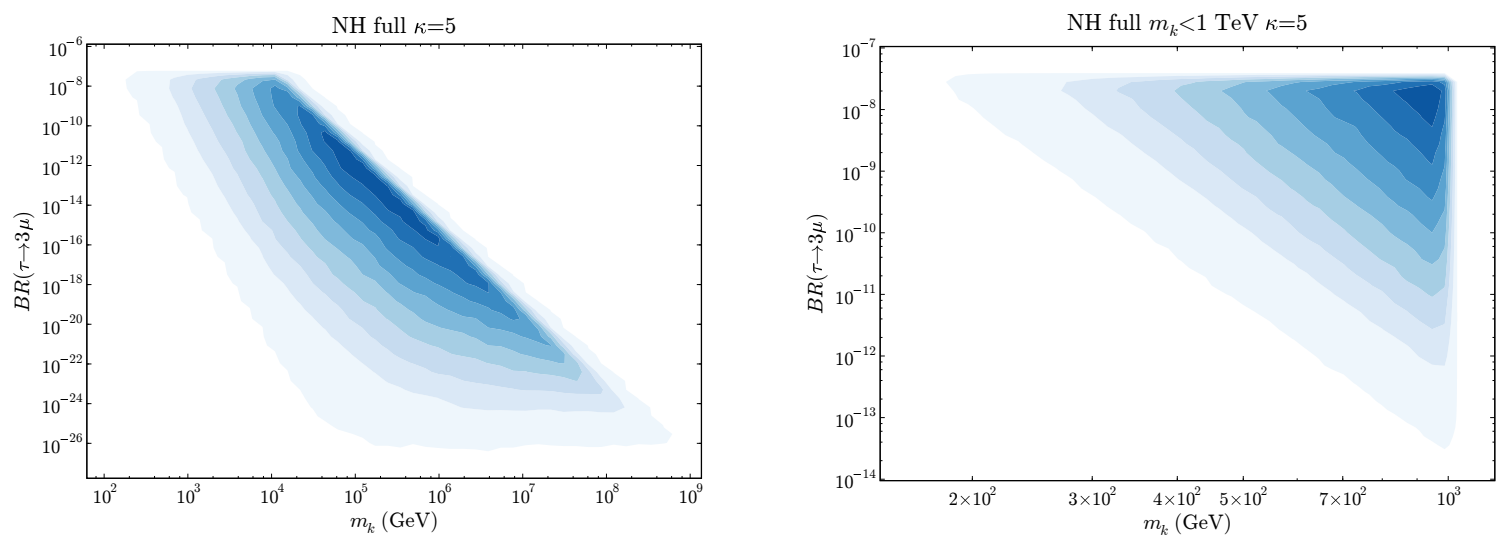

Figure 14: NH: $B R(\tau \rightarrow 3 \mu)$ vs $m_{k}$. 


\section{B. Inverted Hierarchy}

In this section we consider correlations among observables in the inverted hierarchy case.
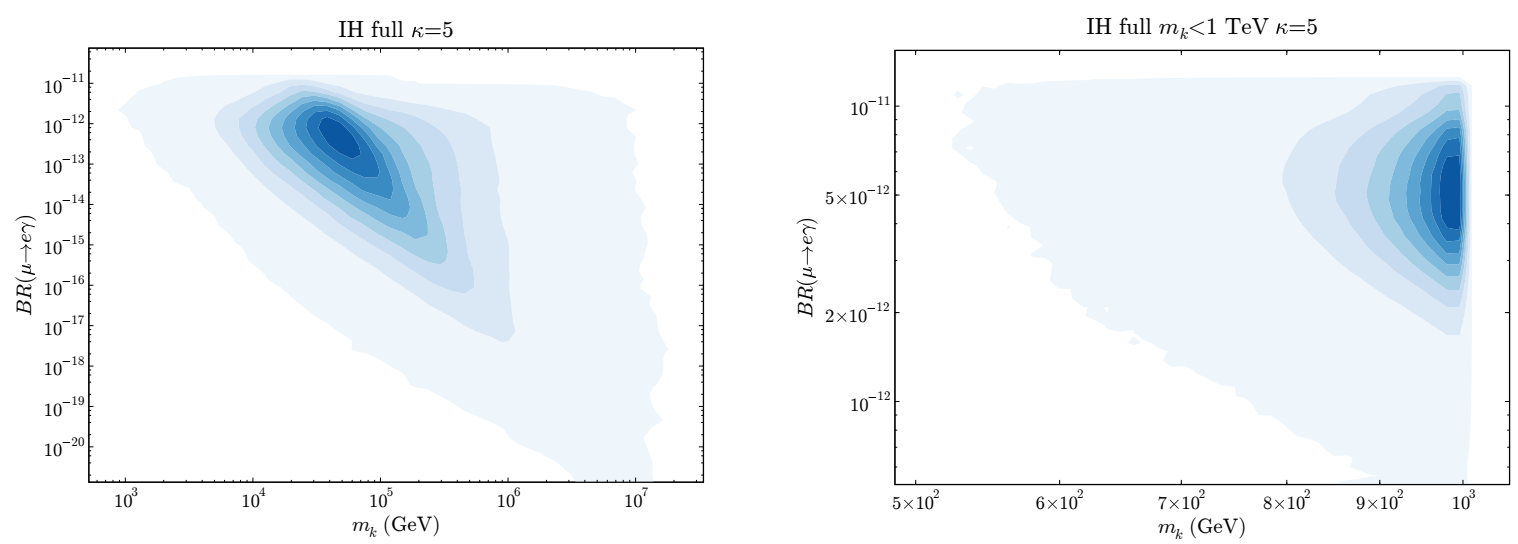

Figure 15: IH: $B R(\mu \rightarrow e \gamma)$ vs $m_{k}$.

In fig. 15 we represent $B R(\mu \rightarrow e \gamma)$ vs $m_{k}$. On the left for the general case and on the right with the additional assumption that the $k^{++}$has been seen at the LHC $\left(m_{k}<1 \mathrm{TeV}\right)$. The plots are similar to the plots obtained in the NH case, although slightly more restrictive. The allowed region in the general case is $m_{k} \sim 10^{2}-10^{7} \mathrm{GeV}$ and $B R(\mu \rightarrow e \gamma) \sim 10^{-22}-10^{-11}$ and higher density values occur for $m_{k} \sim 50 \mathrm{TeV}$ and $B R(\mu \rightarrow e \gamma) \sim 10^{-12}$. However, if nature chooses a $k^{++}$light enough to be produced at the LHC the model is much more constrained: it predicts that $m_{k}$ is relatively large (masses below $400 \mathrm{GeV}$ are only marginally allowed and the preferred masses are above $800 \mathrm{GeV})$. In addition $B R(\mu \rightarrow e \gamma)>10^{-13}$ and the preferred range is above $2 \times 10^{-12}$.

Figure 16 is also similar to fig. 13 but slightly more restrictive. In the general case we find $m_{h} \sim 10^{2}-10^{7} \mathrm{GeV}$ and preferred values $m_{h} \sim 40 \mathrm{TeV}$. For $m_{k}<1 \mathrm{TeV}$ the allowed range of $m_{h}$ is $m_{h} \sim 500 \mathrm{GeV}-70 \mathrm{TeV}$ which will make its detection at the LHC problematic. The constraints on $B R(\tau \rightarrow 3 \mu)$ are also stronger in the IH case, fig. 16, than in the $\mathrm{NH}$ case. The allowed regions are similar but more restrictive. Thus we find that in the general case the preferred values of $B R(\tau \rightarrow 3 \mu)$ are in the $10^{-11}$ range, although values as small as $10^{-21}$ are allowed. In the $m_{k}<1 \mathrm{TeV}$ case the preferred values are $B R(\tau \rightarrow 3 \mu) \gtrsim 3 \times 10^{-9}$ (to be compared with present limits $B R(\tau \rightarrow 3 \mu)<3.2 \times 10^{-8}$ ), but values like $10^{-10}$ are not completely excluded.

Finally, as has been shown analytically, in the IH case there is a lower bound on $\sin ^{2} \theta_{13}$. 

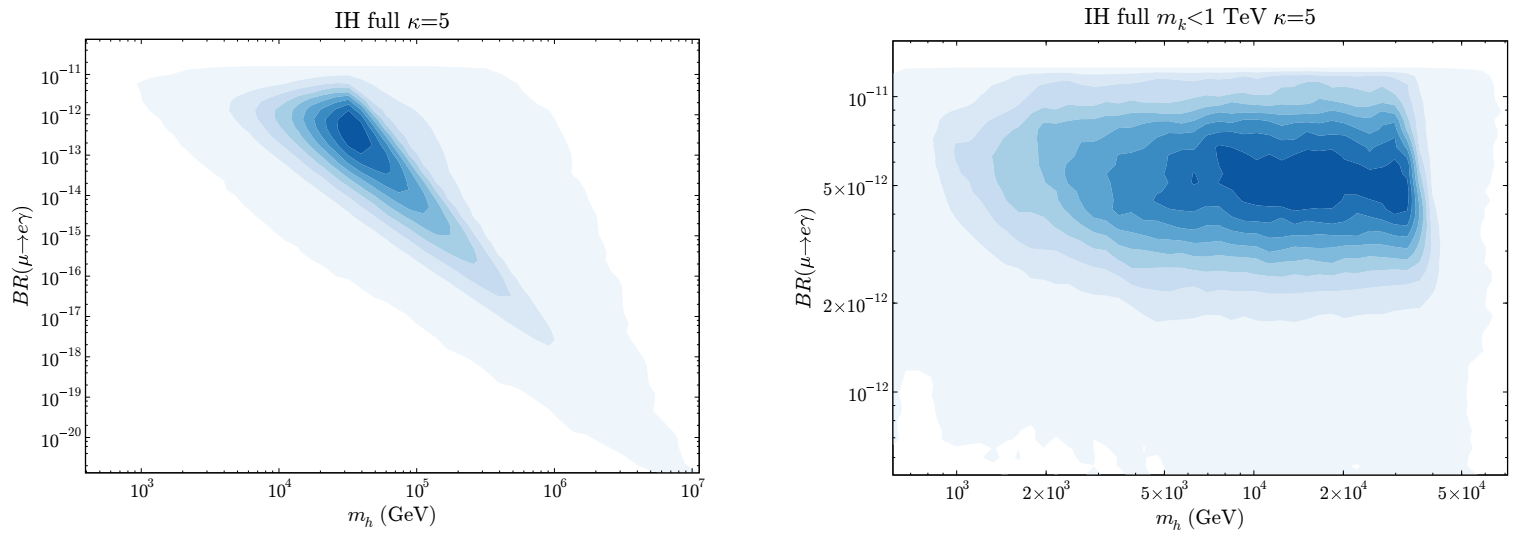

Figure 16: IH: $B R(\mu \rightarrow e \gamma)$ vs $m_{h}$.
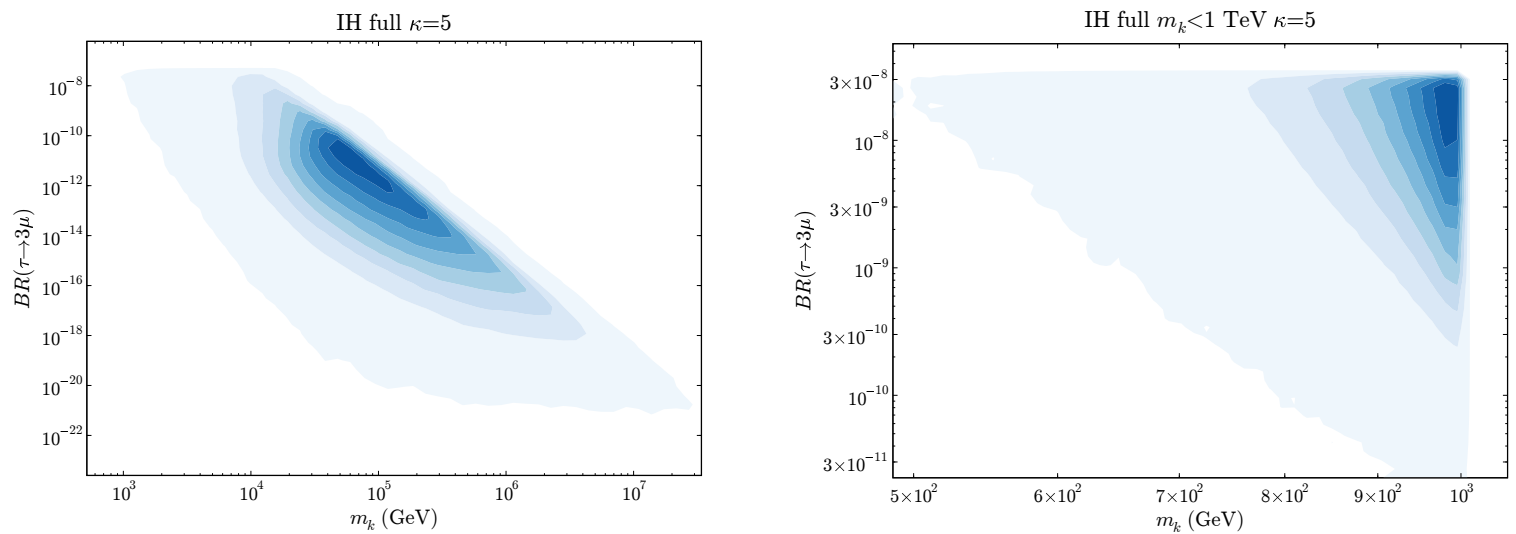

Figure 17: IH: $B R(\tau \rightarrow 3 \mu)$ vs $m_{k}$.

Thus in fig. 18 we represent $\sin ^{2} \theta_{13}$ versus $B R(\mu \rightarrow e \gamma)$. We observe that there is a strong tendency towards relatively large values of $\sin ^{2} \theta_{13}$ even in the general case. The preferred values are in the $\sin ^{2} \theta_{13} \sim 0.01$ region although values below $10^{-4}$ do not seem completely excluded (the present upper limit is $\sin ^{2} \theta_{13}<0.02$ ). In addition smaller values of $\sin ^{2} \theta_{13}$ require larger values of $B R(\mu \rightarrow e \gamma)$. If we also require that the $k^{++}$can be discovered at the LHC we find the preferred values of the model are constrained to a region $\sin ^{2} \theta_{13} \gtrsim 0.01$ and $B R(\mu \rightarrow e \gamma) \gtrsim 10^{-12}$. We also see that values of $\sin ^{2} \theta_{13}$ below 0.005 and $B R(\mu \rightarrow e \gamma)$ below $10^{-13}$ are very unlikely in this case. Notice that mixings as small as $\sin ^{2} \theta_{13} \sim 0.005$ will be tested in a near future [66] and that the MEG experiment, which will start this year, will probe $B R(\mu \rightarrow e \gamma)$ at the level of $10^{-13}$. 

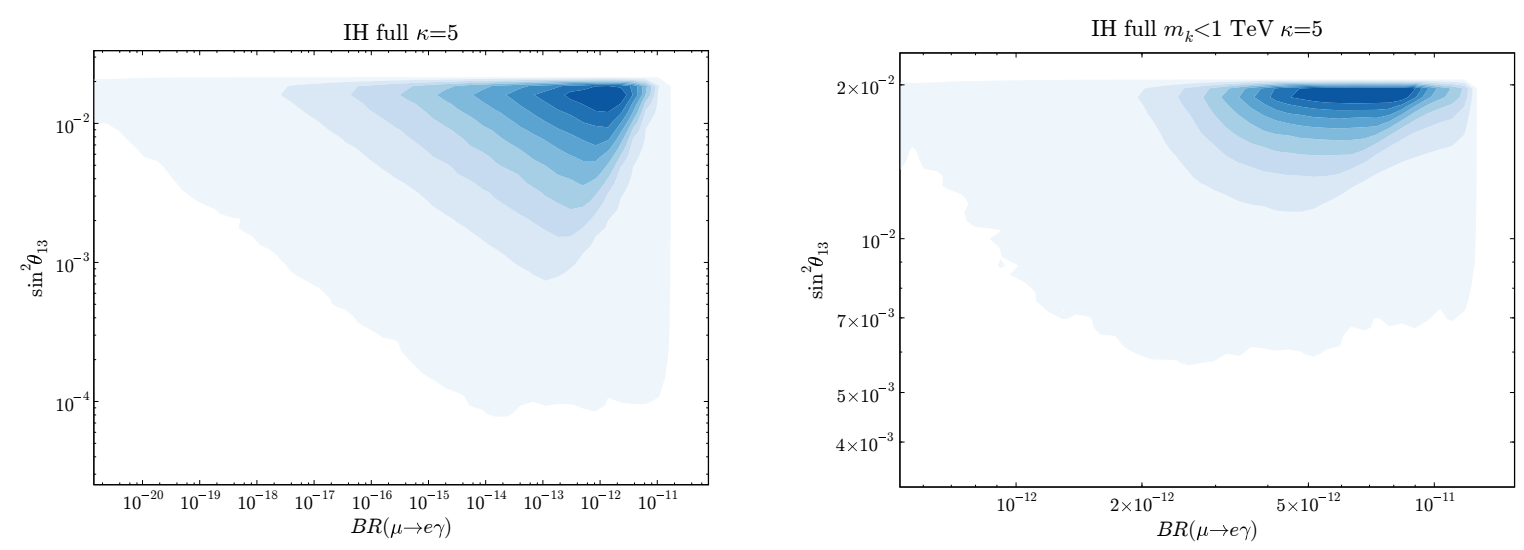

Figure 18: IH: $\sin ^{2} \theta_{13}$ vs $B R(\mu \rightarrow e \gamma)$.

\section{CONCLUSIONS}

In this section we would like to highlight some of the most relevant conclusions drawn from the previous analysis.

The explanation of observed neutrino mixings in the model sets very strong constraints on the structure of the couplings of the singly charged scalars to fermions, in particular, in the NH case the couplings must satisfy $f_{e \tau} \simeq f_{\mu \tau} / 2 \simeq f_{e \mu}$. To fix the absolute value we need another observable, in the NH case we find that the strongest bound comes from $\mu \rightarrow e \gamma$ and tells us that $\left|f_{e i}\right| \lesssim 0.05\left(m_{h} / \mathrm{TeV}\right)$ and $\left|f_{\mu \tau}\right| \lesssim 0.1\left(m_{h} / \mathrm{TeV}\right)$. In the case of IH the couplings must satisfy $f_{e \tau} \simeq-f_{e \mu}$ and $\left|f_{e \tau}\right| \gtrsim 5\left|f_{\mu \tau}\right|$. Then, from lepton-hadron universality we find $\left|f_{e i}\right| \lesssim 0.1\left(m_{h} / \mathrm{TeV}\right)$, and $\left|f_{\mu \tau}\right| \lesssim 0.02\left(m_{h} / \mathrm{TeV}\right)$, bounds which are similar to the bounds obtained from $\mu \rightarrow e \gamma$.

The structure of the couplings of the doubly charged scalar is also very constrained by neutrino masses and mixings. In the case of NH they must satisfy, to a good degree of precision, that $\left|g_{\mu \tau}\right| \approx\left|g_{\mu \mu}\right|\left(m_{\mu} / m_{\tau}\right)$ and $\left|g_{\tau \tau}\right| \approx\left|g_{\mu \mu}\right|\left(m_{\mu} / m_{\tau}\right)^{2}$. In the case of IH this relation does not need to be satisfied exactly because the electron couplings $g_{e i}$ can be relevant. However, we have seen that in a large region of the parameter space this relation is also required. Then, the best constraint comes from $\tau^{-} \rightarrow \mu^{+} \mu^{-} \mu^{-}$which tells us that $\left|g_{\mu \mu}\right| \lesssim 0.4\left(m_{k} / \mathrm{TeV}\right),\left|g_{\mu \tau}\right| \lesssim 0.024\left(m_{k} / \mathrm{TeV}\right),\left|g_{\tau \tau}\right| \lesssim 0.0015\left(m_{k} / \mathrm{TeV}\right)$. The $g_{e i}$ couplings are not constrained by neutrino data but are constrained by low-energy processes which are summarized in tables [ and 【II]

We find that the neutrinoless double beta decay parameter $\left\langle m_{\nu}\right\rangle_{e e}$ is strongly constrained 
in the model. We find $0.001 \mathrm{eV}<\left|\left\langle m_{\nu}\right\rangle_{e e}\right|<0.004 \mathrm{eV}$ in the $\mathrm{NH}$ case and $0.01 \mathrm{eV}<$ $\left|\left\langle m_{\nu}\right\rangle_{e e}\right|<0.06 \mathrm{eV}$ in the IH case. This is just a consequence of the measured neutrino masses and mixings and the particular structure of neutrino masses of the model which predicts a massless neutrino.

If this model is the right explanation for neutrino masses and if $m_{k}<1 \mathrm{TeV}$ the LHC will produce more than 10 events in the $4 l e p$ channel (see section IIII) ${ }^{18}$. There could be some dilution of the signal because the $k$ can also decay into tau leptons or into two singly charged scalars but in the case of $\mathrm{NH}$ this can only be relevant for $m_{k}>1 \mathrm{TeV}$ or if the singly charged scalar is light enough, $m_{k}>2 m_{h}$. In the IH case the dilution of the 4lep signal is a bit larger, still, most of the parameter space with $m_{k}<1 \mathrm{TeV}$ will give more than 10 events in the $4 l e p$ channel as long as the $2 h$ channel is not open.

If more than 10 events are produced at the LHC in the $4 l e p$ channel we find that $B R(\mu \rightarrow$ $e \gamma)>10^{-13}$ in both the $\mathrm{NH}$ and the IH cases. These values are precisely the sensitivity expected in the MEG experiment at the Paul Scherrer Institute (PSI) which will start to run soon [23]. In fact one goal of the experiment is to obtain a significant result before the start of the LHC experiments. If this goal is achieved and nothing is seen we can reverse the argument and claim that it will be very difficult to find the charged scalars of this model at the LHC. We find that if $B R(\mu \rightarrow e \gamma)<10^{-13}$, then, $m_{k}>900 \mathrm{GeV}$ and $m_{h}>600 \mathrm{GeV}$ in the NH case and that both scalar masses will be above the TeV in the case of IH.

It is also important to remark that the photonic vector form factor in muon-electron conversion in nuclei is enhanced with respect to the tensor form factor due to logarithmic corrections of loops with doubly charged scalars. Thus, if the current precision in muonelectron conversion experiments is increased in the next years, there will be additional tests on the model.

If the doubly charged scalars are light enough to be produced at the LHC there are also interesting contributions to rare tau decays. For instance, in the IH case we find that most of the parameter space lies in the region $B R(\tau \rightarrow 3 \mu) \gtrsim 3 \times 10^{-9}$, The NH case allows for slightly smaller branching ratios $B R(\tau \rightarrow 3 \mu) \gtrsim 10^{-9}$. These results have to be compared with the the present limit, $B R(\tau \rightarrow 3 \mu)<3.2 \times 10^{-8}$ or the ranges that might be explored

18 We assume that, once efficiencies are taken into account, this corresponds to 2 reconstructed events [50, 52]. 
in SuperB factories $\sim 10^{-9}-10^{-10}[67,68]$.

The model gives a negative contribution to the $a_{\mu}=\left(g_{\mu}-2\right) / 2$ of the muon. This means it cannot explain a positive deviation from the SM. Conversely, the precise measurements of $a_{\mu}$ set interesting constraints on the parameters of the model.

In general it is much more difficult to satisfy all the constraints in the IH case than in the $\mathrm{NH}$ case. This can be seen in the MC acceptance rate which is much lower in the $\mathrm{IH}$ case than in the NH case. In fact, we have seen that satisfying all the neutrino mass data in the IH case requires certain cancellations in the neutrino mass formulas which imply that the neutrino Majorana phase $\phi$ cannot be zero, actually, most of the parameter space lies in the region $e^{i \phi} \simeq-1$.

Another interesting feature of the $\mathrm{IH}$ case is the emergence of a lower bound on the $\theta_{13}$ mixing. We find that, even in the general case, most of the allowed parameter space requires $\sin ^{2} \theta_{13} \gtrsim 0.001$, although values below $10^{-4}$ do not seem completely excluded. In addition, smaller values of $\sin ^{2} \theta_{13}$ require larger values of $B R(\mu \rightarrow e \gamma)$. If we also require that the $k^{++}$is seen at the LHC through the 4 lep channel we find that values of $\sin ^{2} \theta_{13}$ below 0.005 are very unlikely (the present upper limit is $\sin ^{2} \theta_{13}<0.02$ and mixings as small as $\sin ^{2} \theta_{13} \sim 0.005$ will be tested in a near future [66] ).

In short, the requirement that the model is able to explain the observed pattern of neutrino masses and mixings places very strong limits on the parameters of the model. Thus, if the doubly charged scalar of the model is seen at the LHC through the 4lep channel, the model predicts large contributions to several low energy processes, $\mu \rightarrow e \gamma, \tau \rightarrow 3 \mu$, hadronlepton universality tests, which should be within reach of the next round of experiments. In particular, the MEG, $\mu \rightarrow e \gamma$ experiment [23] should provide results at the required level of precision before the start of the LHC experiments. If the $k^{++}$is discovered at the LHC and MEG does not see anything, the model will be in serious trouble. In all the other cases the model can fit all the data. Moreover, if the $k^{++}$is discovered at the LHC and MEG sees $\mu \rightarrow e \gamma$ in the allowed region, the model will be a serious candidate to explain neutrino masses. 


\section{Acknowledgments}

This work has been supported in part by the MEC (Spain) under the grant FPA200500711 and by the EU MRTN-CT-2006-035482 (FLAVIAnet). M.N. acknowledges financial support from "Fundação para a Ciência e a Tecnologia" (FCT, Portugal) through the projects PDCT/FP/63912/2005, PDCT/FP/63914/2005, CFTP-FCT UNIT 777, and by the EU MRTN-CT-2006-035505 (HEPtools).

[1] J. Davis, Raymond, D.S. Harmer and K.C. Hoffman, Phys. Rev. Lett. 20 (1968) 1205.

[2] KAMIOKANDE-II, K.S. Hirata et al., Phys. Lett. B205 (1988) 416.

[3] B.T. Cleveland et al., Astrophys. J. 496 (1998) 505.

[4] GALlEX, W. Hampel et al., Phys. Lett. B447 (1999) 127.

[5] GNO, M. Altmann et al., Phys. Lett. B490 (2000) 16, hep-ex/0006034.

[6] SNO, Q.R. Ahmad et al., Phys. Rev. Lett. 89 (2002) 011301, nucl-ex/0204008.

[7] KamLAND, K. Eguchi et al., Phys. Rev. Lett. 90 (2003) 021802, hep-ex/0212021.

[8] Super-Kamiokande, Y. Fukuda et al., Phys. Rev. Lett. 81 (1998) 1562, hep-ex/9807003.

[9] Soudan-2, W.W.M. Allison et al., Phys. Lett. B449 (1999) 137, hep-ex/9901024.

[10] K2K, E. Aliu et al., Phys. Rev. Lett. 94 (2005) 081802, hep-ex/0411038.

[11] J.R. Ellis et al., Phys. Lett. B150 (1985) 142.

[12] A. Santamaria and J.W.F. Valle, Phys. Lett. B195 (1987) 423.

[13] L.J. Hall and M. Suzuki, Nucl. Phys. B231 (1984) 419.

[14] M. Hirsch et al., Phys. Rev. D62 (2000) 113008, hep-ph/0004115.

[15] G.B. Gelmini and M. Roncadelli, Phys. Lett. B99 (1981) 411.

[16] H.M. Georgi, S.L. Glashow and S. Nussinov, Nucl. Phys. B193 (1981) 297.

[17] A. Zee, Phys. Lett. B93 (1980) 389.

[18] A. Zee, Phys. Lett. B161 (1985) 141.

[19] A. Zee, Nucl. Phys. B264 (1986) 99.

[20] K.S. Babu, Phys. Lett. B203 (1988) 132.

[21] S. Bertolini and A. Santamaria, Nucl. Phys. B310 (1988) 714.

[22] A. Santamaria, Phys. Rev. D39 (1989) 2715. 
[23] MEG, S. Ritt, Nucl. Phys. Proc. Suppl. 162 (2006) 279.

[24] Y. Koide, Phys. Rev. D64 (2001) 077301, hep-ph/0104226.

[25] K.R.S. Balaji, W. Grimus and T. Schwetz, Phys. Lett. B508 (2001) 301, hep-ph/0104035.

[26] J.F. Oliver and A. Santamaria, Phys. Rev. D65 (2002) 033003, hep-ph/0108020.

[27] K.S. Babu and C. Macesanu, Phys. Rev. D67 (2003) 073010, hep-ph/0212058.

[28] D. Aristizabal Sierra and M. Hirsch, JHEP 12 (2006) 052, hep-ph/0609307.

[29] Belle, K. Abe et al., hep-ex/0609049,

Belle, Y. Miyazaki et al., arXiv:0711.2189 [hep-ex].

[30] Belle, Y. Yusa et al., Phys. Lett. B589 (2004) 103, hep-ex/0403039;

Belle, K. Hayasaka et al., arXiv:0705.0650 [hep-ex].

[31] BaBar, F.F. Wilson, Nucl. Phys. Proc. Suppl. 162 (2006) 181;

BaBar, B. Aubert et al., Phys. Rev. Lett. 99 (2007) 251803, arXiv:0708.3650 [hep-ex];

BaBar, B. Aubert et al., Phys. Rev. Lett. 96 (2006) 041801, hep-ex/0508012;

BaBar, B. Aubert et al., Phys. Rev. Lett. 95 (2005) 041802, hep-ex/0502032.

[32] A. Santamaria, Phys. Lett. B305 (1993) 90, hep-ph/9302301.

[33] K.L. McDonald and B.H.J. McKellar, , hep-ph/0309270.

[34] J. Bernabeu, A. Pich and A. Santamaria, Z. Phys. C30 (1986) 213.

[35] K. Horikawa and K. Sasaki, Phys. Rev. D53 (1996) 560, hep-ph/9504218.

[36] L. Willmann et al., Phys. Rev. Lett. 82 (1999) 49, hep-ex/9807011.

[37] Particle Data Group, W.M. Yao et al., J. Phys. G33 (2006) 1.

[38] A. Pich, Int. J. Mod. Phys. A21 (2006) 5652, hep-ph/0609138.

[39] S.M. Bilenky and S.T. Petcov, Rev. Mod. Phys. 59 (1987) 671.

[40] A. Pich, A. Santamaria and J. Bernabeu, Phys. Lett. B148 (1984) 229.

[41] M. Raidal and A. Santamaria, Phys. Lett. B421 (1998) 250, hep-ph/9710389.

[42] S.R. Moore, K. Whisnant and B.L. Young, Phys. Rev. D31 (1985) 105.

[43] J.P. Leveille, Nucl. Phys. B137 (1978) 63.

[44] M. Passera, J. Phys. G31 (2005) R75, hep-ph/0411168.

[45] D.A. Dicus, H.J. He and J.N. Ng, Phys. Rev. Lett. 87 (2001) 111803, hep-ph/0103126.

[46] T. Kinoshita, Nucl. Phys. Proc. Suppl. 157 (2006) 101.

[47] G.J. Feldman and R.D. Cousins, Phys. Rev. D57 (1998) 3873, physics/9711021.

[48] J.F. Gunion et al., Phys. Rev. D40 (1989) 1546. 
[49] K. Huitu et al., Nucl. Phys. B487 (1997) 27, hep-ph/9606311.

[50] J.F. Gunion, C. Loomis and K.T. Pitts, ECONF C960625 (1996) LTH096, hep-ph/9610237.

[51] A.G. Akeroyd and M. Aoki, Phys. Rev. D72 (2005) 035011, hep-ph/0506176.

[52] G. Azuelos, K. Benslama and J. Ferland, J. Phys. G32 (2006) 73, hep-ph/0503096.

[53] B. Dion et al., Phys. Rev. D59 (1999) 075006, hep-ph/9810534.

[54] F. Cuypers and S. Davidson, Eur. Phys. J. C2 (1998) 503, hep-ph/9609487.

[55] DELPHI, J. Abdallah et al., Phys. Lett. B552 (2003) 127, hep-ex/0303026.

[56] OPAL, G. Abbiendi et al., Phys. Lett. B526 (2002) 221, hep-ex/0111059.

[57] L3, P. Achard et al., Phys. Lett. B576 (2003) 18, hep-ex/0309076.

[58] OPAL, G. Abbiendi et al., Phys. Lett. B577 (2003) 93, hep-ex/0308052.

[59] D0, V.M. Abazov et al., Phys. Rev. Lett. 93 (2004) 141801, hep-ex/0404015.

[60] CDF, D. Acosta et al., Phys. Rev. Lett. 93 (2004) 221802, hep-ex/0406073.

[61] CDF, D. Acosta et al., Phys. Rev. Lett. 95 (2005) 071801, hep-ex/0503004.

[62] A. Pukhov et al., CompHEP: A package for evaluation of Feynman diagrams and integration over multi-particle phase space. User's manual for version 33, 1999, hep-ph/9908288.

[63] D. Stump et al., JHEP 10 (2003) 046, hep-ph/0303013.

[64] M. Muhlleitner and M. Spira, Phys. Rev. D68 (2003) 117701, hep-ph/0305288.

[65] T. Schwetz, Phys. Scripta T127 (2006) 1, hep-ph/0606060.

[66] P. Huber et al., JHEP 05 (2006) 072, hep-ph/0601266.

[67] SuperKEKB Physics Working Group, A.G. Akeroyd et al., hep-ex/0406071.

[68] M. Bona et al., arXiv:0709.0451 [hep-ex]. 